\title{
PROBE DETECTION OF PHASED EEDFS IN RF DISCHARGES
}

\author{
Francis F. Chen \\ Electrical Engineering Department
}

PPG- 1542

July, 1995 


\title{
DC Probe Detection of Phased EEDFs in RF Discharges
}

\author{
Francis F. Chen
}

Electrical Engineering Department, University of California, Los Angeles, CA 90024-1594

\begin{abstract}
Detection of non-Maxwellian electron energy distribution functions (EEDFs) by Langmuir probes in the presence of radiofrequency (RF) fluctuations in plasma potential is possible if the probe is properly RFcompensated. If the non-thermal electrons are created by wave acceleration, however, they usually are bunched at one phase of the RF. In this case, the usual method of forcing the probe to follow fluctuations in floating potential $\left(V_{\mathrm{f}}\right)$ will be inappropriate, since $V_{\mathrm{f}}$ itself will be time-dependent in such a way as to ensure that the fast electron tail is not detected. The probe must be made to follow fluctuations in space potential. Computations are given to show the effect of RF on distributions with high-energy tails with and without the proper compensation. Singular EEDFs arising in the case of constant energy gain are also treated for the first time.
\end{abstract}




\section{INTRODUCTION}

In some RF plasma sources, such as the helicon source [1], it has been suggested that the ionizing electrons could be accelerated by wave-particle interactions [2]. In this case, the fast electrons, of the order of 50-100 eV, would be bunched in the accelerating phase of the wave's parallel electric field $E_{z}$, where $z$ is the direction of the DC magnetic field $\mathbf{B}_{0}$, as shown in Fig. 1. The occurrence of such bunched electrons has been inferred from the observed time variation of optical light emission in synchronism with the RF [3]. To detect these electrons with a simple diagnostic such as a Langmuir probe requires careful RF compensation to remove the nonlinear averaging imposed by large RF fluctuations in plasma potential. Many methods for doing this can be found in the literature on RF plasma sources. For instance, the method developed by Sudit and Chen [4] employs RF chokes to filter the fundamental and first harmonic of the RF frequency and, in addition, a large auxiliary floating electrode to sense the voltage fluctuations near the probe tip and to couple them to the tip, causing it to follow the fluctuations. Unfortunately, the floating potential itself is affected by the fast electrons, in such a way that $V_{\mathrm{f}}$ shifts negatively whenever the fast electrons are collected. The result is that the RF compensation is too strong and guarantees that the electron tails cannot be seen on the probe characteristics. This is true of any RF probe method which relies on feeding back a floating potential signal. An ideal probe would sense the local space potential $V_{\mathrm{s}}$ and use that for RF feedback, thus avoiding the self-masking aspect of phased beams. At this time we do not know of an easy way to do this.

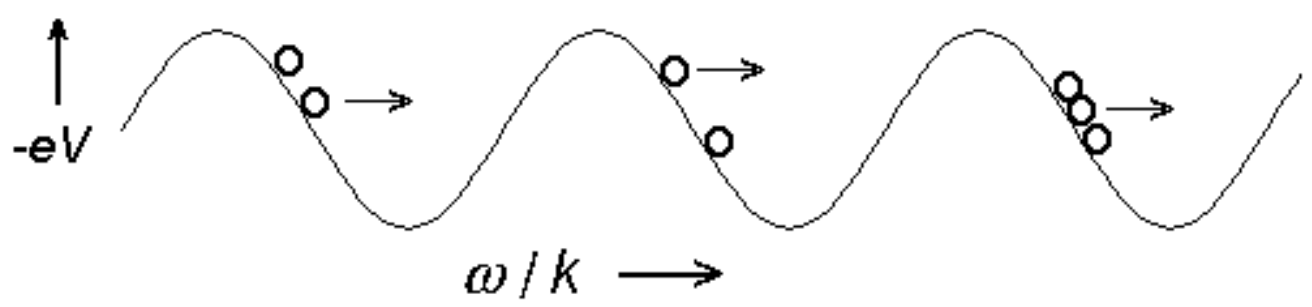

Fig. 1. The electric potential seen by electrons, showing their bunching at the phase of a propagating wave at which there is an accelerating electric field.

In this paper, we compute the probe characteristics for typical discharges with various levels of RF noise and with various fast electron tails, phased and unphased. The effects of insufficient, perfect, and excessive RF compensation are calculated. These current-voltage (I- 
V) curves are similar to those shown previously by others [5] but are somewhat more realistic in that the rounded "knee" observed in the presence of strong magnetic fields is reproduced in the computations. In addition, a distribution of fast electrons other than a shifted Maxwellian is also treated; this is the case of singular EEDFs which arise if the energy gain of the fast electrons, rather than their velocity gain, is constant. Finally, we show experimental tests of our conclusions. The ideas conveyed here are general enough to be applicable to any RF discharge in which the electron distribution function varies at the RF frequency. Though we have implied the existence of a magnetic field in order to use a simple one-dimensional electron distribution, this simplification is not essential.

\section{FORMULATION}

Let $V_{\mathrm{p}}$ be the probe bias voltage and $V_{\mathrm{s}}$ the space, or plasma, potential. We neglect oscillations in plasma density and temperature but allow $V_{\mathrm{s}}$ to vary sinusoidally at the RF frequency:

$$
V_{s}=V_{r f} \cos \omega t
$$

The probe potential relative to the plasma is defined as $V$ :

$$
V \equiv V_{p}-V_{s}
$$

The minimum velocity of an electron that can reach the probe is then given by

$$
1 / 2 m v_{m}^{2}=-e V
$$

Hence,

$$
v_{m}=(-2 e V / m)^{1 / 2}, \quad v_{m}^{2} / v_{e}^{2}=-e V / K T_{e} .
$$

Let the bulk plasma electrons be Maxwellian with temperature $T_{\mathrm{e}}$, and let the ions be cold. We consider the one-dimensional case, in which the normalized electron velocity distribution is

where

$$
f_{e}(v)=\left(1 / v_{e} \sqrt{\pi}\right) \exp \left(-v^{2} / v_{e}^{2}\right)
$$

$$
v_{e} \equiv\left(2 K T_{e} / m\right)^{1 / 2}, \quad \bar{v}_{e} \equiv v_{e} / 2 \sqrt{\pi}
$$


Added to this is a population of fast (beam) electrons of density $n_{\mathrm{b}}$, whose distribution will be discussed later. The densities at the sheath edge are related by

$$
n_{i}=n_{e}+n_{b}
$$

\section{Thermal electron current}

For $V<0$, the thermal electron flux collected by the probe is

$$
j_{e}=n_{e} \varlimsup_{v_{m}}^{\infty} f_{e}(v) d v=\left(n_{e} v_{e} / 2 \sqrt{\pi}\right) \exp \left(-v_{m}^{2} / v_{e}^{2}\right)=n_{e} \overline{v_{e}} \exp \left(e V / K T_{e}\right)
$$

Defining the dimensionless variables

$$
J=j / n_{e} \bar{v}_{e}, \quad \eta=e V / K T_{e}
$$

we can write Eq. (8) as

$$
J_{e}=e^{\eta}
$$

For $V>0, J_{\mathrm{e}}$ is replaced by its saturation value $J_{\text {sat }}$. In a strong magnetic field, the probe's presence necessarily changes the plasma potential on the flux tube that it blocks, and saturation starts well before $V_{\mathrm{p}}-V_{\mathrm{s}}=0$, if $V_{\mathrm{s}}$ is referred to the plasma on undisturbed flux tubes. For the purposes of this paper, it is sufficient to model the saturation current with a two-parameter curve (a parabola) that resembles the $I$ - $V$ characteristics seen experimentally. Let $a$ be the value of $J_{\mathrm{e}}$ at which saturation begins, and let $b$ describe the rate at which the saturation current grows. Matching the slope of the parabola to that of Eq. (10) at $J_{\mathrm{e}}=a$, we obtain

$$
J_{\text {sat }}=a-b^{2} / 2 a+b\left[\eta-\ln a+(b / 2 a)^{2}\right]^{1 / 2} .
$$

The potential $\eta_{\mathrm{c}}$ at the onset of saturation is given by $\eta_{\mathrm{c}}=\ln a$. Thus, the thermal electron current is given by

$$
\begin{array}{ll}
J_{e}=e^{\eta} & \eta<\eta_{c} \\
J_{e}=J_{s a t} & \eta>\eta_{c}
\end{array}
$$




\section{Ion current}

The normalized saturation ion current is approximately [5]

$$
J_{i} \approx 0.5 \frac{n_{e}+n_{b}}{n_{e} \bar{v}_{e}} \overline{F_{M}} \frac{T_{e}}{M} K^{1 / 2}=(1+f) \overline{\frac{\pi}{2} m} \frac{m}{M} \mathbf{K}^{1 / 2},
$$

where $\mathrm{M}$ is the ion mass. In practice, the ion current varies slowly with voltage and can be approximated by a parabola. Since the exact ion current is not critical here, we arbitrarily set it to have the value in Eq. (13) at a probe potential of $-36 K T_{\mathrm{e}}$. Thus we take the normalized ion current to be

$$
J_{i}=(1+f) \sqrt{\frac{\pi}{2} m} K_{M}^{1 / 2} \frac{\sqrt{-\eta}}{6}
$$

\section{Electron beam current}

The normalized probe current $J_{b}$ due to accelerated "beam" electrons will be derived in the next section, giving a total probe current

$$
J=J_{e}+J_{b}-J_{i}
$$

\section{BEAM DISTRIBUTIONS}

The distribution function of the non-thermal electrons depends on the acceleration mechanism. We assume that the accelerated electrons are concentrated at the phase of the RF at which the electric field has the proper sign and is at its maximum so that its magnitude is insensitive to phase. A runaway population of these electrons can be defined as those which are quickly accelerated well above their thermal speeds before making a collision. These electrons will experience the electric field until they fall out of phase or make a collision with an ion or a neutral atom. We assume that the collisional mechanism prevails. If their collision cross section varies as $1 / v$, then the collision frequency will be constant, and the electrons will, on average, be accelerated for a time equal to $1 / n_{\mathrm{o}} \sigma v$, where $n_{\mathrm{o}}$ is the density of scattering centers. In that case, the velocity gained by each electron will be the same regardless of its initial velocity, and the EEDF is that of a shifted Maxwellian, the case usually treated. On the other hand, if the cross section is constant, the electrons will, on average, be accelerated for a 
given distance; namely, the mean free path $\lambda_{\mathrm{m}}=1 / n_{\mathrm{o}} \sigma$. This distance is approximately constant for a distribution of about $3 \mathrm{eV}$ width accelerated to 50-100 eV. In this case, the energy gained by each electron will be the same, leading to a different EEDF. We have calculated the probe currents for these two extremes; the actual distribution will probably lie in between. One-dimensional distributions are used, since the magnetic field has been assumed strong enough that even the beam electrons move primarily in the $z$ direction.

\section{1) Shifted Maxwellian}

At the sheath edge, the distribution function of a Maxwellian of temperature $T_{\mathrm{b}}$ shifted by a velocity $u$ is:

$$
\begin{gathered}
f_{b}(v)=\left(1 / v_{b} \sqrt{\pi}\right) \exp \left[-(v-u)^{2} / v_{b}^{2}\right], \text { where } \\
v_{b} \equiv\left(2 K T_{b} / m\right)^{1 / 2}, \quad \bar{v}_{b} \equiv v_{b} / 2 \sqrt{\pi} .
\end{gathered}
$$

The beam current is then

$$
j_{b}=\frac{n_{b}}{v_{b} \sqrt{\pi}} \bigotimes_{m} \exp \left[-(v-u)^{2} / v_{b}^{2}\right] v d v
$$

This yields

$$
j_{b}=n_{b} \bar{v}_{b}\left[\exp \left(-y_{m}^{2} / v_{b}^{2}\right)+\left(u / v_{b}\right) \sqrt{\pi} \operatorname{erfc}\left(y_{m} / v_{b}\right)\right]
$$

where $y_{\mathrm{m}}=v_{\mathrm{m}}-u$ [Eq. (4)], $\operatorname{erfc}(x)=1-\operatorname{erf}(x)=1+\operatorname{erf}(-x)$, and

$$
\operatorname{erf}(x)=\frac{2}{\sqrt{\pi}} \mathbb{Z}^{-t^{2}} d t
$$

In terms of the dimensionless quantities

$$
f \equiv n_{b} / n_{e}, \quad h \equiv v_{b} / v_{e}, \quad q \equiv u / v_{b},
$$

the normalized beam current can be written

$$
J_{b}=j_{b} / n_{e} \bar{v}_{e}=f h\left[\exp \left(-Y_{m}^{2}\right)+q \sqrt{\pi} \operatorname{erfc}\left(Y_{m}\right)\right]
$$

where

$$
\begin{aligned}
Y_{m} & =y_{m} / v_{b}=(-\eta)^{1 / 2} / h-q & & \eta<0 \\
& =-q & & \eta>0
\end{aligned}
$$


This assumes that the fast electrons saturate above the space potential. The initial and final distributions are sketched in Fig. 2; the "final" distribution is that found at the sheath edge, before it is changed by the sheath electric field.

\section{2) Constant energy case}

In this case, let the beam distribution start as a Maxwellian at temperature $T_{\mathrm{b}}$, and let each accelerated electron gain or lose an energy $W$. The center of the velocity distribution will then be shifted by an amount $v_{\mathrm{c}}$, where

$$
v_{c}=(2 W / m)^{1 / 2} \text {. }
$$

The initial distribution is divided into three regions, as shown in Fig. 3. Those electrons originally moving in the direction of the acceleration will gain an energy $\mathrm{W}$; those originally moving fast in the opposite direction will lose an energy $\mathrm{W}$; and those moving slowly in the backward direction will be turned around. The final velocity $v$ of an electron with initial velocity $v_{\mathrm{O}}$ is thus given by

$$
\begin{aligned}
& \text { Region 1) } \quad v_{\mathrm{o}}>0: \quad v^{2}=v_{\mathrm{o}}^{2}+v_{\mathrm{c}}^{2} \quad(v>0) \\
& \left(\text { Region 2) }-v_{\mathrm{c}}<v_{\mathrm{O}}<0: \quad v^{2}=v_{\mathrm{c}}^{2-} v_{\mathrm{O}}^{2} \quad(v>0)\right. \\
& \left(\text { Region 3) } \quad v_{\mathrm{o}}<-v_{\mathrm{c}}: \quad v^{2}=v_{\mathrm{o}}^{2}-v_{\mathrm{c}}^{2} \quad(v<0) .\right.
\end{aligned}
$$

These regions, shown in Fig. 3, map into the corresponding regions of the accelerated distribution in Fig. 4.

Region 1. We have

$$
v_{0}^{2}=v^{2}-v_{c}^{2}, \quad d v_{0}=\left(v / v_{0}\right) d v .
$$

Taking

$$
f_{0}\left(v_{0}\right) d v_{0}=f(v) d v
$$

with $f_{0}\left(v_{0}\right)$ given by Eq. (16), we obtain

$$
f(v)=\frac{1}{v_{b} \sqrt{\pi}} \frac{v}{\left(v^{2}-v_{c}^{2}\right)^{1 / 2}} \exp \left[-\left(v^{2}-v_{c}^{2}\right) / v_{b}^{2}\right]
$$


Note that $f(v)$ has an integrable singularity at $v=v_{\mathrm{c}}$. That accelerated distributions can be singular is well known [6]. The physical reason for this is that $f_{0}\left(v_{0}\right)$ has zero slope at $v_{0}$, so that these particles, which end up at $v=v_{\mathrm{c}}$, are bunched into an infinitesimally small velocity interval. Similarly, the Jacobean dictates that $f(v)$ has to vanish at $v=0$. Integrating $f(v)$ from $v_{\mathrm{c}}$ to $\infty$, we find that the fraction of particles in this region is $1 / 2$, as expected.

Region 2. Now we have

$$
v_{0}^{2}=v_{c}^{2}-v^{2}, \quad d v_{0}=-\left(v / v_{0}\right) d v .
$$

This gives

$$
f(v)=\frac{1}{v_{b} \sqrt{\pi}} \frac{v}{\left(v_{c}^{2}-v^{2}\right)^{1 / 2}} \exp \left[-\left(v_{c}^{2}-v^{2}\right) / v_{b}^{2}\right] .
$$

The fraction of particles in this region is $1 / 2 \operatorname{erf}\left(v_{\mathrm{c}} / v_{\mathrm{b}}\right)$.

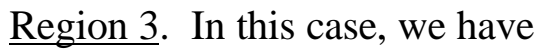

$$
v_{0}^{2}=v_{c}^{2}+v^{2}, \quad d v_{0}=\left(v / v_{0}\right) d v,
$$

so that

$$
f(v)=-\frac{1}{v_{b} \sqrt{\pi}} \frac{v}{\left(v^{2}+v_{c}^{2}\right)^{1 / 2}} \exp \left[-\left(v^{2}+v_{c}^{2}\right) / v_{b}^{2}\right] .
$$

The fraction of particles in this region is $1 / 2 \operatorname{erfc}\left(v_{\mathrm{c}} / v_{\mathrm{b}}\right)$, where erfc is the complementary error function. Fig. 5 shows $f(v)$ for various values of $W$, and Fig. 6 traces how the zero and pole in $f(v)$ develop as a Maxwellian distribution is given a small energy gain.

The current of fast electrons to the probe is given by

$$
j_{b}=n_{b} \bar{Z}_{m} v f(v) d v
$$

If $v_{\mathrm{m}}$ is larger than $v_{\mathrm{c}}$, the integral lies within Region 1 , and $f(v)$ is given by Eq. (26). The normalized collected beam current is then

$$
\left.J_{b}=\frac{f h}{\sqrt{\pi}} \sum_{Y_{m}}^{\infty} Z y^{2}+q^{2}\right)^{1 / 2} \exp \left(-y^{2}\right) d y, \quad\left(v_{m}>v_{c}\right)
$$

where

$$
y=\left(v^{2}-v_{c}^{2}\right)^{1 / 2} / v_{b}, \quad Y_{m}=\left(v_{m}^{2}-v_{c}^{2}\right)^{1 / 2} / v_{b}=\left(-\eta / h^{2}-q^{2}\right)^{1 / 2} .
$$


If $v_{\mathrm{m}}$ is smaller than $v_{\mathrm{c}}$, part of the distribution lies in Region 2, and Eq. (28) has to be used for that part. The result is

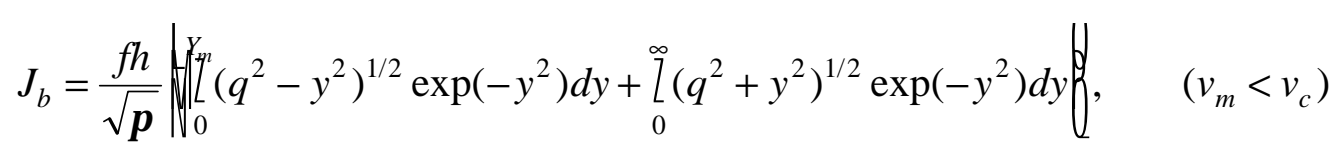

where, in the first integral,

$$
y=\left(v_{c}^{2}-v^{2}\right)^{1 / 2} / v_{b}, \quad Y_{m}=\left(v_{c}^{2}-v_{m}^{2}\right)^{1 / 2} / v_{b}=\left(\eta / h^{2}+q^{2}\right)^{1 / 2} .
$$

In this case, saturation occurs for $\eta>0$, and $Y_{\mathrm{m}}$ has a maximum value of $q$.

\section{Recovery of $f(v)$}

For three-dimensional distributions, $f(v)$ is proportional to the second derivative of the probe current; but in strong magnetic fields, the electron motion is one-dimensional, and $f(v)$ is given by the first derivative:

$$
j_{e}=n_{e} \prod_{v_{m}}^{\infty} f(v) d v, \quad d j_{e} / d v_{m}=-n_{e} v_{m} f\left(v_{m}\right), \quad d j_{e} / d V=n_{e}(e / m) f\left(v_{m}\right),
$$

where Eq. (4) was used for $v_{\mathrm{m}}$. In terms of the dimensionless quantities $J$ and $\eta$ [Eq. (9)], we have

$$
f\left(v_{m}\right)=\frac{1}{v_{e} \sqrt{\pi}} \frac{d J}{d \eta}
$$

For a shifted Maxwellian, Eq. (22) gives

$$
d J_{b} / d \eta=f h^{-1} \exp \left[-(\sqrt{-\eta} / h-q)^{2}\right] .
$$

Though the maximum of this curve occurs at the expected potential, the e-folding points of the curve are separated by $\Delta \eta=4 q h^{2}$, a factor $q \gg 1$ larger than the width of $2 h^{2}$ of the original energy distribution.. The beam distribution, as it would appear when plotted against probe potential, is shown in Fig. 7 for various beam temperatures and velocity shifts. The distribution seems to widen as it is accelerated, but this is because $\partial J / \partial \eta \approx f(v)$ is plotted against $v^{2}, \operatorname{not} v$.

For the constant-energy case, the peak of the beam occurs at the probe potential corresponding to the energy gain (Fig. 8), but the width of the beam narrows (in velocity 
space) as $\mathrm{W}$ is increased (Fig. 5). Since $f(v)$ is no longer Gaussian, the width of the observed $f(v)$ depends on how well the singularity is resolved. With good resolution, a probe might yield the $f(v)$ curve shown in Fig. (8), which was computed from Eqs. (33), (35), and (38) with a grid size $\Delta \eta=0.1$. The width appears to be less than $T_{b}=1 \mathrm{eV}$, illustrating the well known effect of acceleration cooling. A coarser grid or probe resolution would give, however, a wider apparent $f(v)$. Figure 9 compares the apparent $f(v)$ 's for two types of beam, one with constant-velocity acceleration and the other with constant-energy acceleration, both starting with the same temperature $T_{\mathrm{b}}$ and suffering the same mean energy gain $E_{\mathrm{b}}$. It is clear that the apparent widths can be very different.

\section{COMPUTED PROBE CURVES}

In this section we use the formulas of Secs. II and III for computations illustrating the effects of RF fluctuations, fast electron populations, phased beams, and $V_{\mathrm{f}}$-compensation on probe $I-V$ curves. To permit the ion saturation current to be seen, we have increased it by choosing a light ion, namely $\mathrm{He}$, in all the calculations. We have also simplified the calculation by choosing a shifted Maxwellian for the beam electrons.

\section{Effect of RF pickup on dc beams}

Fig. 10a shows the model probe curve given by Eqs. (15), (12), (14), and (22). Fig. $10 \mathrm{~b}$ shows how the I-V curve, averaged over an RF cycle, is distorted by varying magnitudes of $V_{\text {rf. }}$ This behavior is in general agreement with results published earlier by Hershkowitz [5] and others. In practice, the RF potential fluctuation in the plasma can be as large as several hundred volts; in this case, $V_{\mathrm{rf}}$ represents the amount of RF pickup remaining after attempts to eliminate it. Fig. 10c shows the apparent velocity distribution $f(v)$ obtained by differentiating the curves in Fig. 10b. Even $5 \mathrm{~V}$ of uncompensated $V_{\text {rf }}$ is sufficient to distort the Maxwellian distribution beyond recognition. In Fig. 11, the effect of adding a dc beam of varying density $n_{\mathrm{b}}$, temperature $T_{\mathrm{b}}$, and center energy $E_{\mathrm{b}}$ is shown. In Fig. 12a, the effect of RF on a probe curve with a dc beam is shown. The apparent $f(v)$ 's for the thermal part of the distribution is indistinguishable from Fig. 10c, but the high-energy portion (Fig. 12b) shows the presence of the beam with the correct central energy even with $V_{\mathrm{rf}}$ as large as $20 \mathrm{~V}$. 


\section{Phased beams}

We now consider electron beams which occur only during the accelerating phase of the RF cycle. Fig. 13a shows the assumed time variation of the beam, and Fig. 13b the probe characteristics for various values of the maximum beam density. Fig. 14a shows the effect of RF pickup on one of these cases, and Fig. 14b the corresponding distribution functions derived therefrom. Note that the beam can be detected as long as $V_{\mathrm{rf}}$ is less than $E_{\mathrm{b}}$.

\section{Effect of $V_{f}$ compensation}

Fig. 15a shows the time-averaged characteristic for a probe in a plasma with a phased beam in the absence of RF pickup, but with compensation circuits designed to follow fluctuations in floating potential. This curve was computed by shifting the $I-V$ curve at each RF phase so that the floating potential occurred at the same probe potential as it did when the beam current was zero. The derivative of this curve gives the apparent distribution function shown in Fig. 15b. We see that the oscillation in floating potential causes $f(v)$ to be smeared out, as if there had been RF pickup. Fig. 16 compares these curves with the ideal curves which would be obtained in the absence of both RF and RF compensation, or in the presence of RF pickup but with ideal compensation following the fluctuations in space potential $V_{\mathrm{s}}$. In Fig. 17a we compare the curve of Fig. 15a with the curve obtained with 20V of uncompensated RF. Fig. $17 \mathrm{~b}$ shows the derived distribution functions. Since the characteristic has already been distorted by $V_{\mathrm{f}}$ compensation, the further addition of RF pickup has only the effect of shifting the curve. Finally, in Fig. 18, we compare the probe characteristics with RF pickup and a phased beam for the cases of no compensation, $V_{\mathrm{f}}$ compensation, and ideal $V_{\mathrm{s}}$ compensation. We see that the presence of the beam cannot be detected with $V_{\mathrm{f}}$ compensation. 


\section{ACKNOWLEDGMENTS}

This work was supported by the National Science Foundation, Grant No. ECS-9400849; the Semiconductor Research Corp., Project 95-IJ-529; the Wisconsin Engineering Research Center for Plasma-Aided Manufacturing, and the Plasma Physics Research Institute of the Lawrence Livermore Laboratory.

\section{REFERENCES}

[1] F.F. Chen and G. Chevalier, J. Vac. Sci. Technol. A 10, 1389 (1992).

[2] F.F. Chen, Plasma Phys. Controlled Fusion 33, 339 (1991).

[3] A.R. Ellingboe, R.W. Boswell, J.P. Booth, and N. Sadeghi, Phys. Plasmas 2, 1807 (1995).

[4] I.D. Sudit and F.F. Chen, Plasma Sources Sci. Technol. 3, 162 (1994).

[5] N. Hershkowitz, in Plasma Diagnostics, Vol. 1, ed. by O. Auciello and D.L. Flamm (Academic Press, 1989), Chap. 3.

[6] L. Tonks and I. Langmuir, Phys. Rev. 34, 876 (1929). 


\section{FIGURE CAPTIONS}

Fig. 1. The electric potential seen by electrons, showing their bunching at the phase of a propagating wave at which there is an accelerating electric field.

Fig. 2. The beam distribution functions before and after wave acceleration, for the case of constant velocity gain. Electrons faster than $v_{\mathrm{m}}$ are able to overcome the probe bias.

Fig. 3. Velocity regions of a Maxwellian beam distribution before each electron is given an energy $W=m v_{\mathrm{c}}^{2} / 2$.

Fig. 4. The beam distribution after acceleration in the constant-energy case. The numbered regions correspond to those in Fig. 3. Electrons with $v>v_{\mathrm{m}}$ reach the probe.

Fig. 5. Velocity distribution of beam electrons in the case of constant energy gain $W$, for various values of $W / K T_{\mathrm{b}}$.

Fig. 6. Velocity distribution of beam electrons for small energy gains, showing the development of the singularity in $f(v)$.

Fig. 7. Apparent velocity distributions of electrons in a shifted Maxwellian for (a) various beam temperatures and (b) various velocity shifts. These curves are obtained by differentiating the probe $I-V$ curves and are therefore plots of $f(v)$ vs. $v^{2}$.

Fig. 8. Apparent velocity distribution of electrons accelerated by a constant energy $W$. This curve is obtained by differentiating the probe $I-V$ curve and are therefore the apparent width depends on the resolution of the computation.

Fig. 9. Apparent velocity distributions of a $1-\mathrm{eV}$ electron distribution, after acceleration by the same mean energy, in the constant-velocity and constant-energy cases. The remarks under Figs. 7 and 8 apply.

Fig. 10. (a) Normal $I-V$ characteristic for a $T_{\mathrm{e}}=3 \mathrm{eV}, T_{\mathrm{i}}=0$ Maxwellian plasma in a magnetic field. (b) The $I-V$ curve of (a) after distortion by RF oscillations of magnitude $V_{\mathrm{rf}}$. (c) Derived velocity distribution $f(v)$ vs. normalized probe voltage.

Fig. 11. (a) $\mathrm{I}-\mathrm{V}$ characteristics for a $3-\mathrm{eV}$ plasma with a $T_{\mathrm{b}}=1 \mathrm{eV}$ beam of various densities. (b) Apparent distributions $f(v)$ derived from (a). (c) Apparent $f(v)$ 's for a plasma with 50$\mathrm{eV}$ beams of various temperatures. The $I-V$ curves from which these were derived are not shown. (d) Apparent $f(v)$ 's for a plasma with beams of the same density but different energies.

Fig. 12. (a) Probe characteristics for a plasma with a dc beam after distortion by various amounts of RF pickup. (b) Apparent distribution function in the region of the beam obtained by differentiation of the curves in (a). 
Fig. 13. (a) Time variation of phased electron beams used in the calculations. (b) Probe characteristics for a 3-eV plasma with a phased beam of various peak densities in the absence of RF pickup and of RF compensation circuitry.

Fig. 14. (a) Uncompensated probe characteristics for a 3-eV plasma with a phased beam and various levels of RF pickup. (b) Apparent distribution functions derived from (a). The rapid oscillations are due to the coarseness of the numerical grid and should be ignored.

Fig. 15. (a) $I-V$ characteristic of a $V_{\mathrm{f}}$ - compensated probe in a $3-\mathrm{eV}$ plasma with a pulsed, 50$\mathrm{eV}$ electron beam. (b) Apparent $f(v)$ from the derivative of (a).

Fig. 16. (a) $I-V$ curves for probes driven to follow $V_{\mathrm{s}}$ and $V_{\mathrm{f}}$ in a plasma with a phased beam. (b) Apparent $f(v)$ 's derived from (a).

Fig. 17. (a) $I-V$ characteristics for $V_{\mathrm{f}}$-compensated probes in a plasma with a phased beam, with $V_{\mathrm{rf}}=0$ and $20 \mathrm{~V}$. (b) Apparent $f(v)$ 's derived from (a).

Fig. 18. $I-V$ characteristics for a probe in a plasma with a phased beam and $V_{\mathrm{rf}}=20 \mathrm{~V}$, for cases of no RF compensation, $V_{\mathrm{f}}$-compensation, and ideal $V_{\mathrm{s}}$-compensation. 


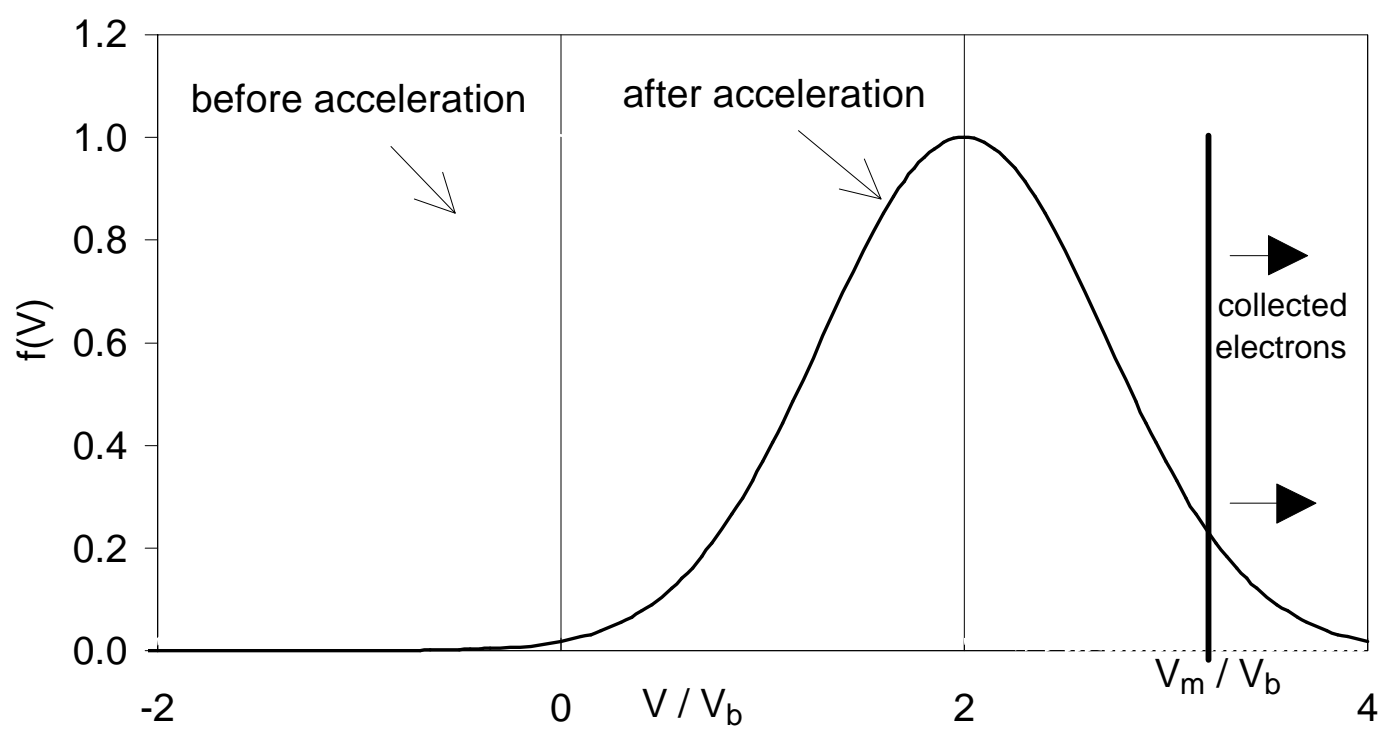

Fig. 2. The beam distribution functions before and after wave acceleration, for the case of constant velocity gain. Electrons faster than $v_{\mathrm{m}}$ are able to overcome the probe bias.

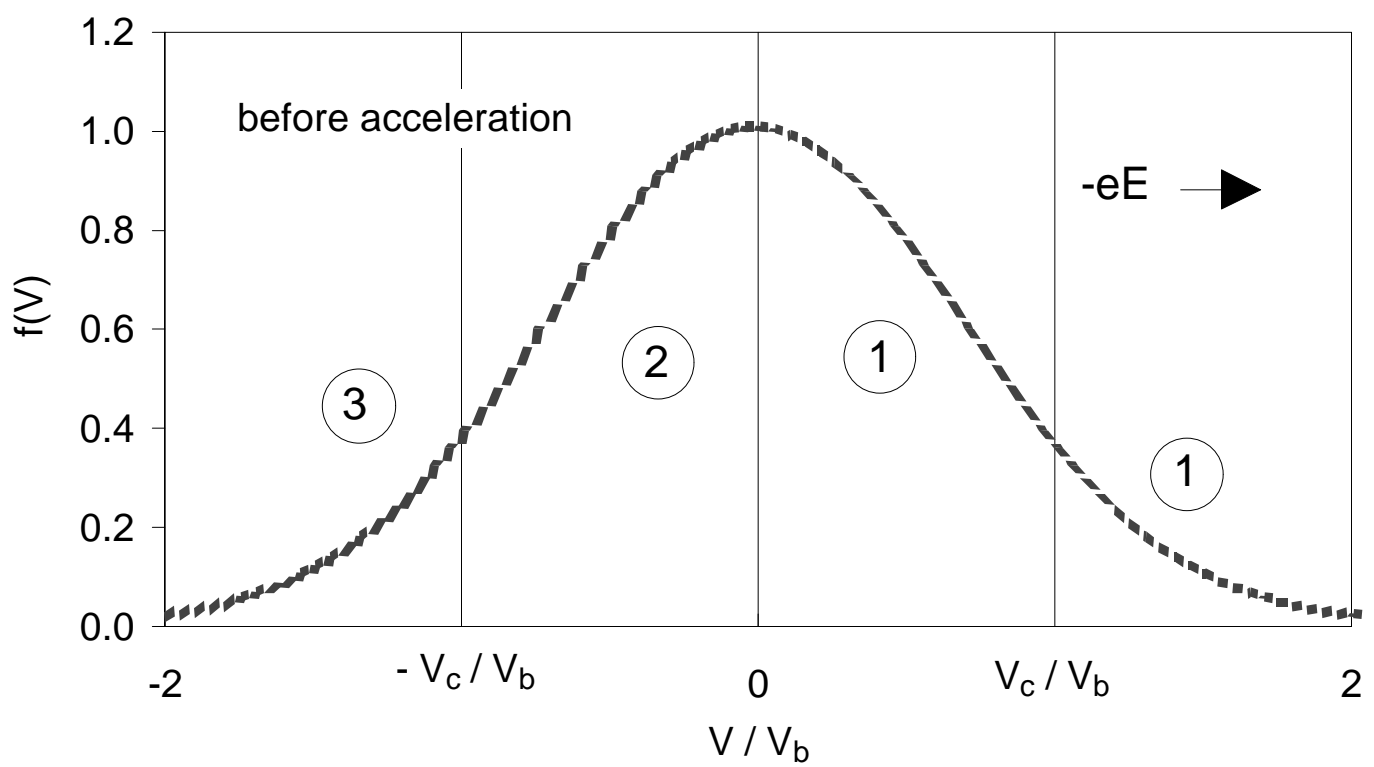

Fig. 3. Velocity regions of a Maxwellian beam distribution before each electron is given an energy $W=m v_{\mathrm{c}}^{2} / 2$. 


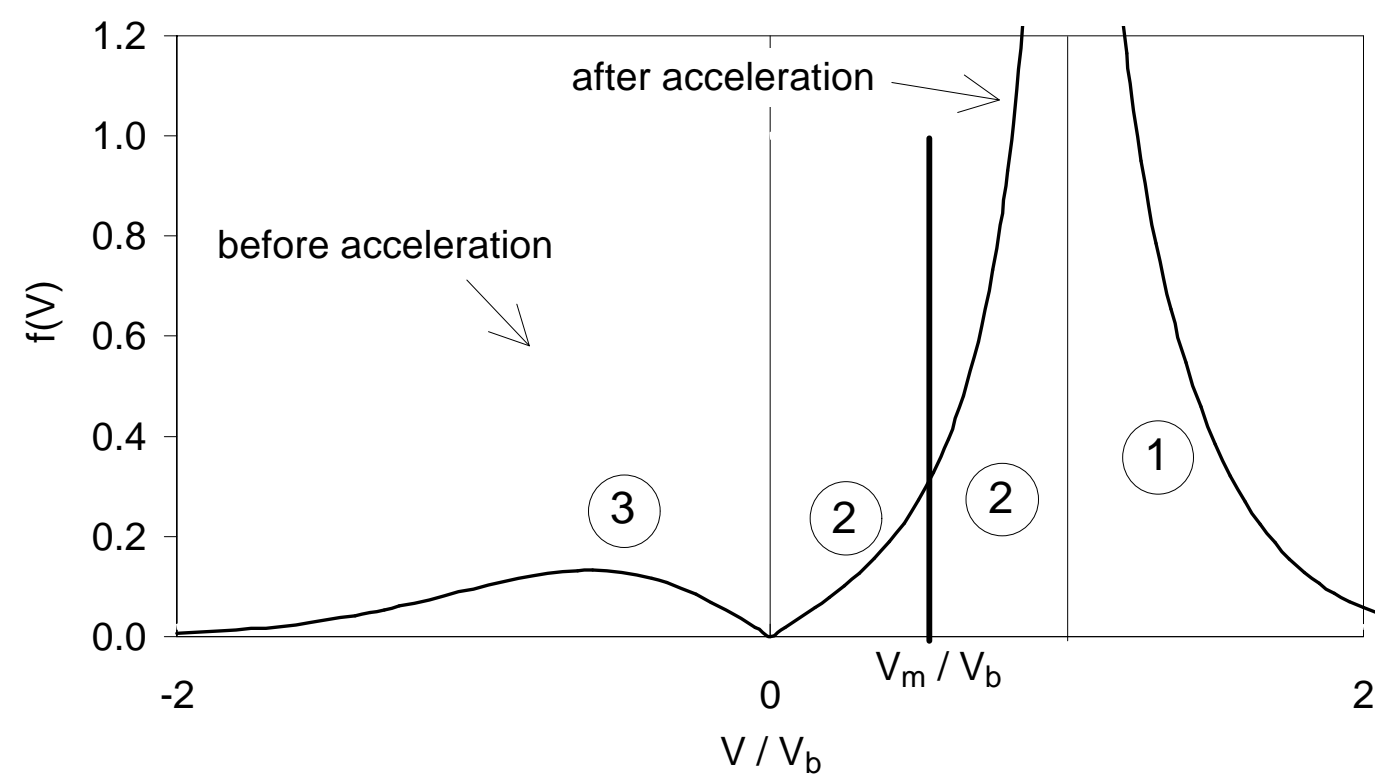

Fig. 4. The beam distribution after acceleration in the constant-energy case. The numbered regions correspond to those in Fig. 3. Electrons with $v>v_{\mathrm{m}}$ reach the probe.

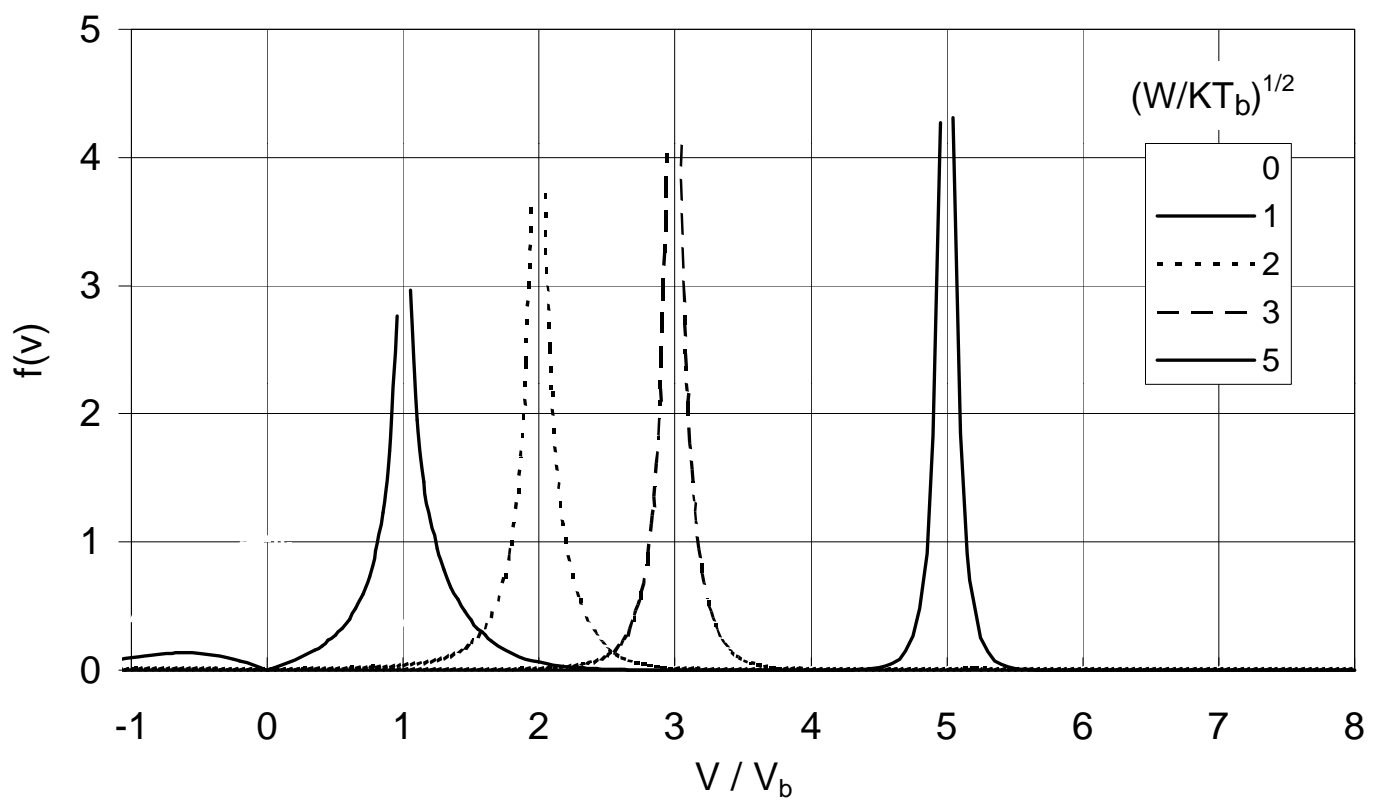

Fig. 5. Velocity distribution of beam electrons in the case of constant energy gain $W$, for various values of $W / K T_{\mathrm{b}}$. 


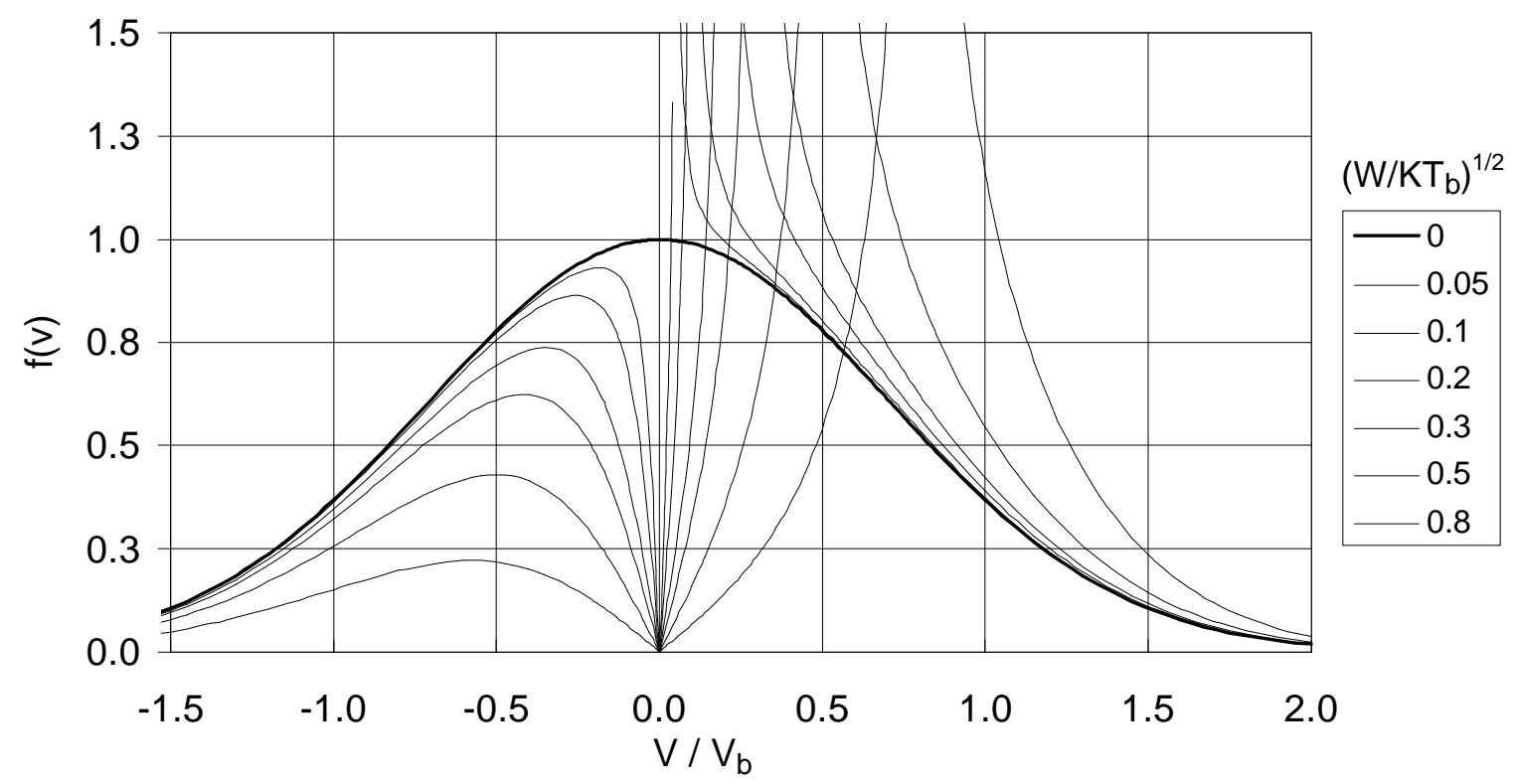

Fig. 6. Velocity distribution of beam electrons for small energy gains, showing the development of the singularity in $f(v)$. 


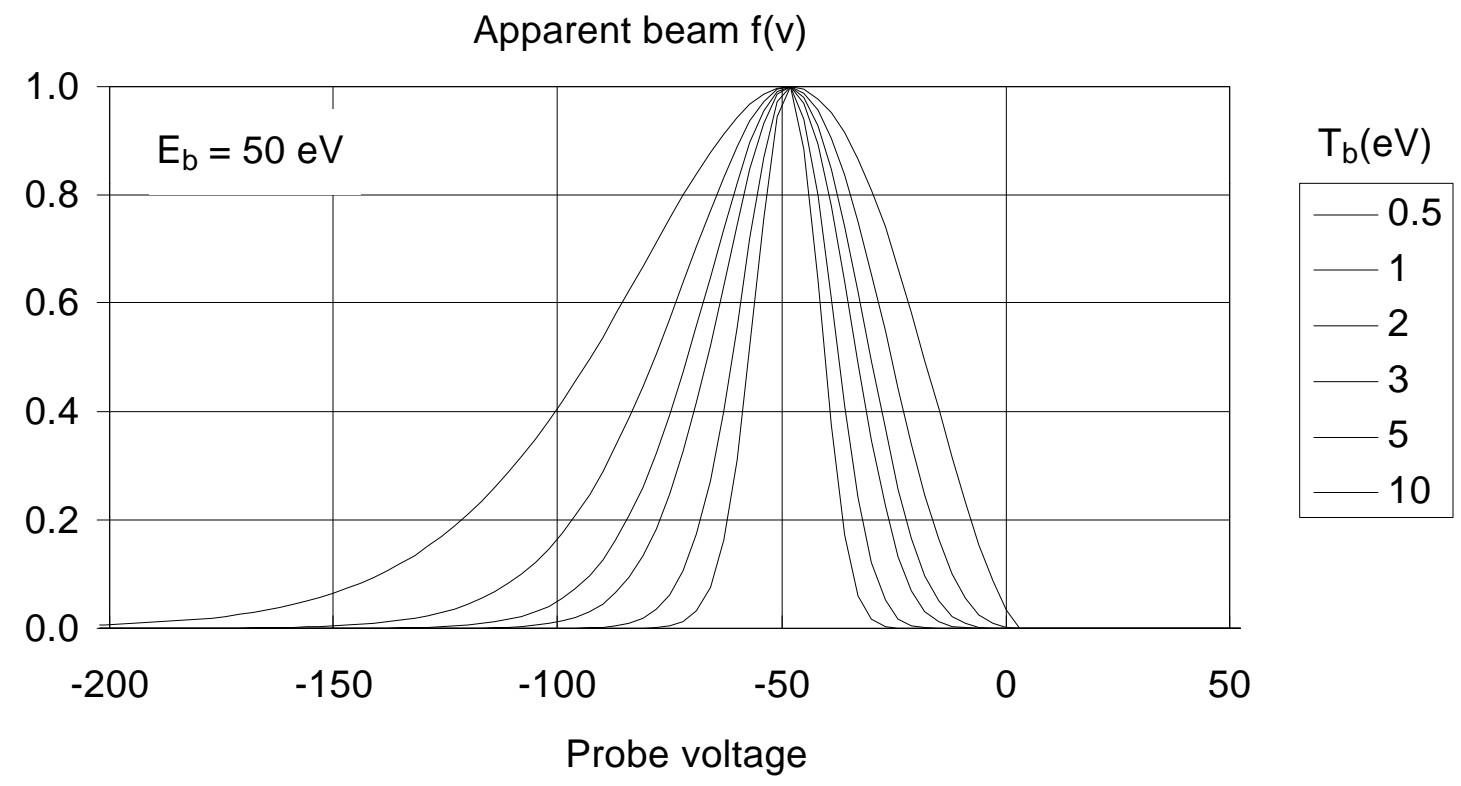

(a)

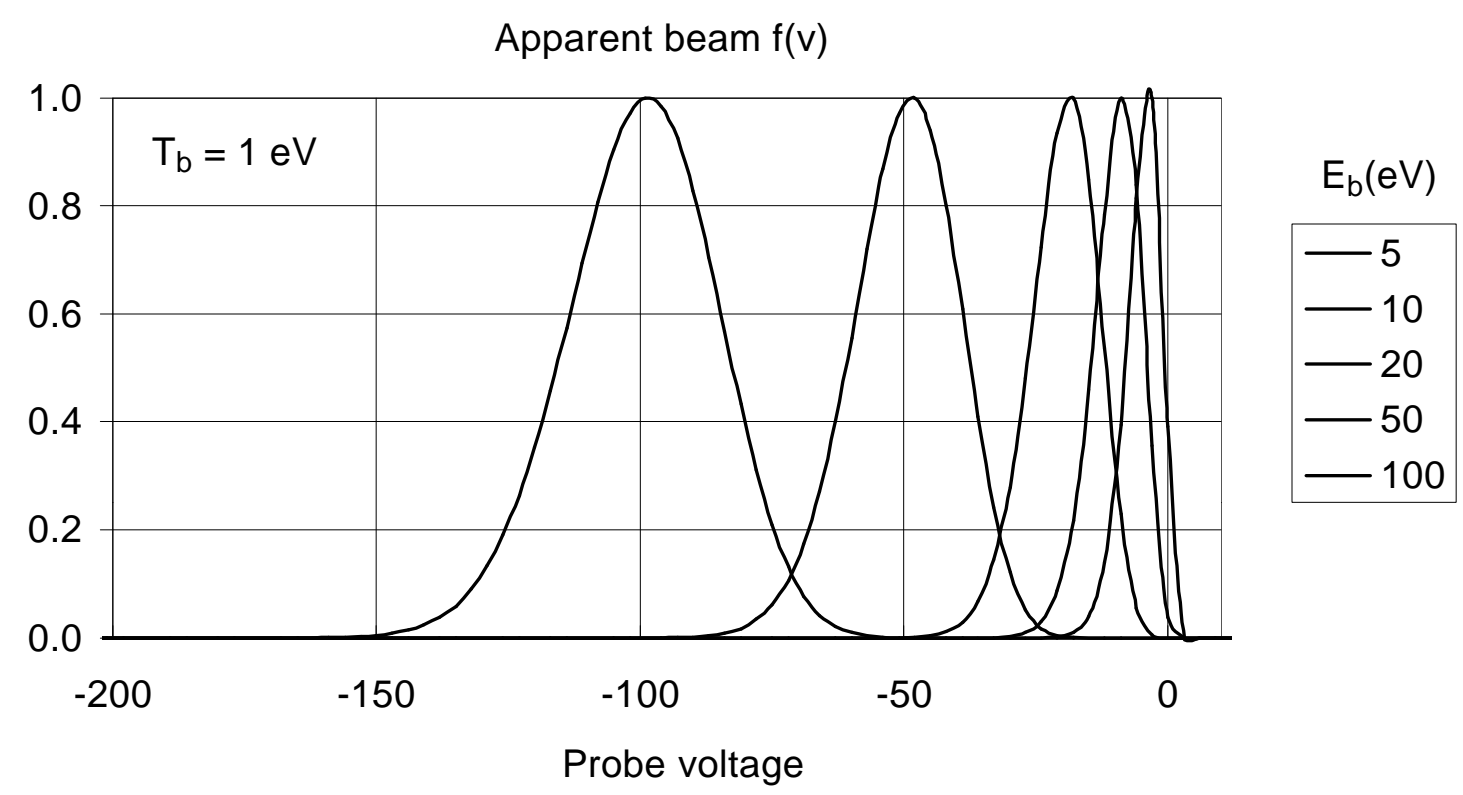

(b)

Fig. 7. Apparent velocity distributions of electrons in a shifted Maxwellian for (a) various beam temperatures and (b) various velocity shifts. These curves are obtained by differentiating the probe $I-V$ curves and are therefore plots of $f(v)$ vs. $v^{2}$. 


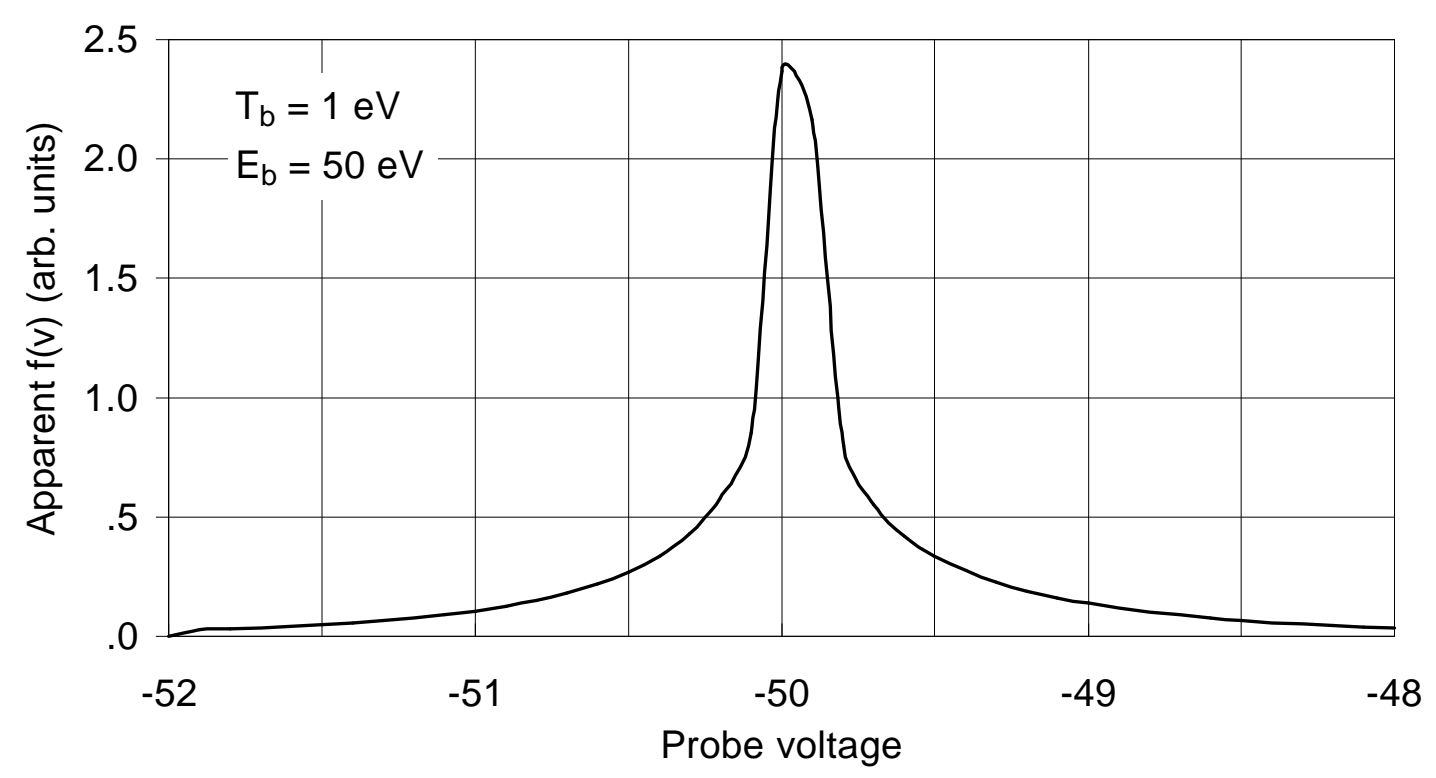

Fig. 8. Apparent velocity distribution of electrons accelerated by a constant energy $W$. This curve is obtained by differentiating the probe $I-V$ curve and are therefore the apparent width depends on the resolution of the computation.

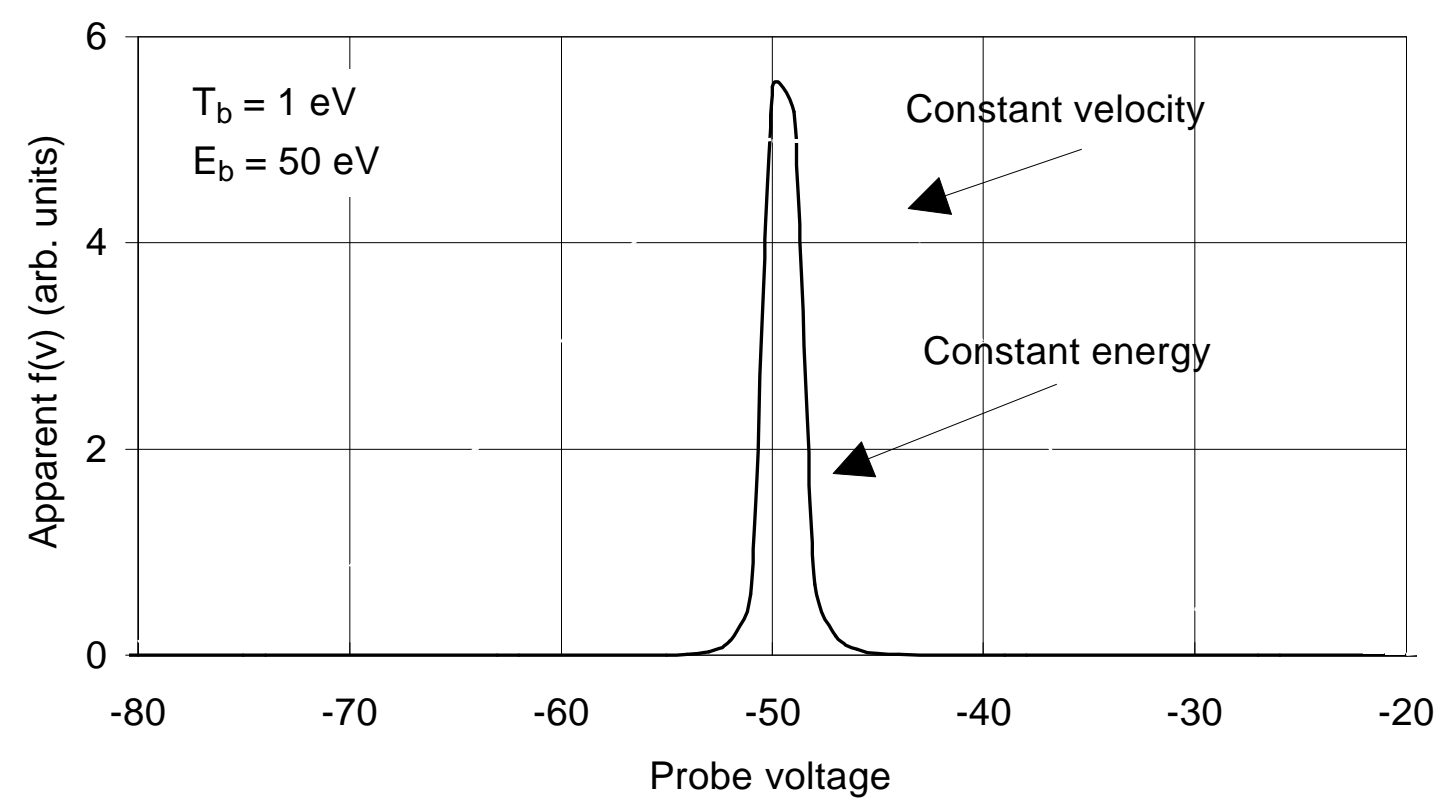

Fig. 9. Apparent velocity distributions of a 1-eV electron distribution, after acceleration by the same mean energy, in the constant-velocity and constantenergy cases. The remarks under Figs. 7 and 8 apply. 


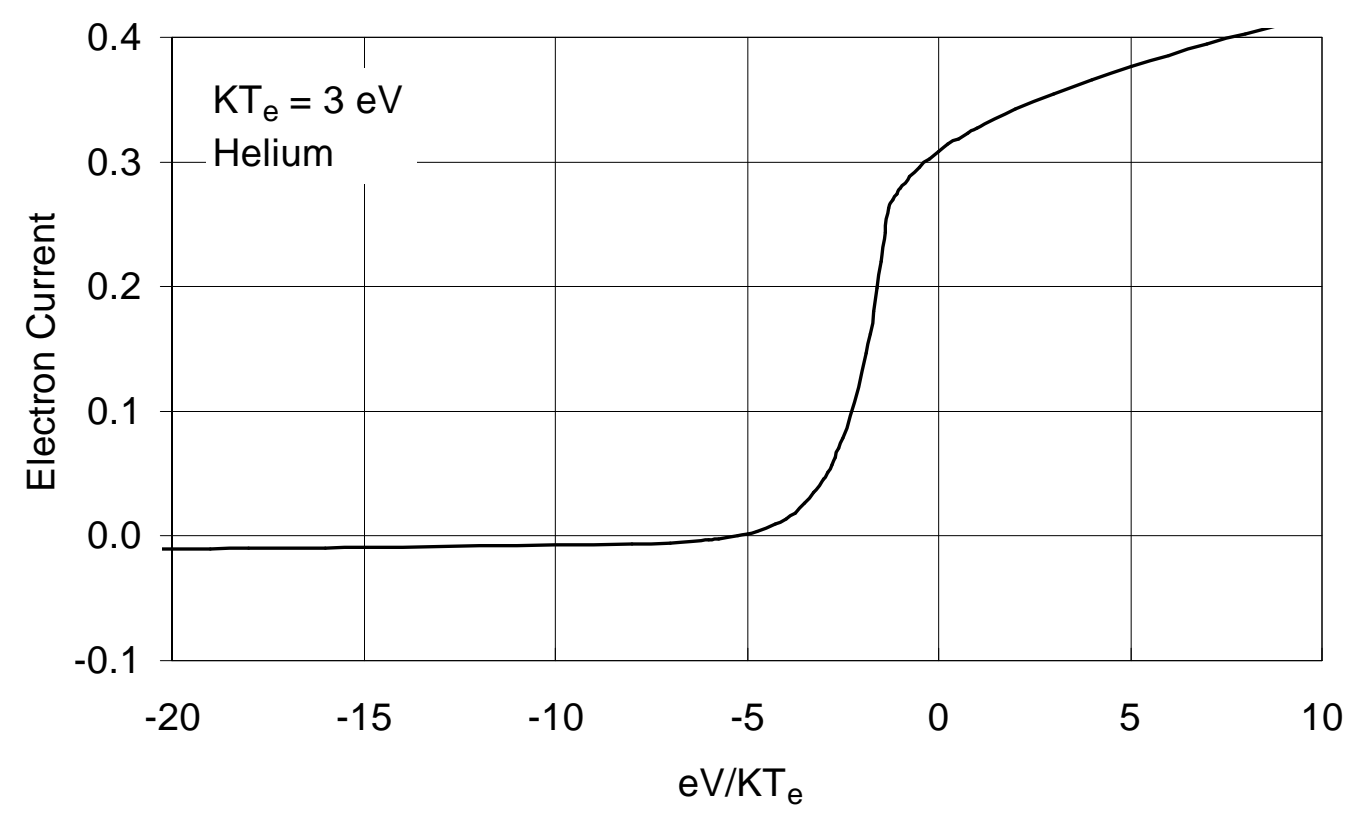

(a)

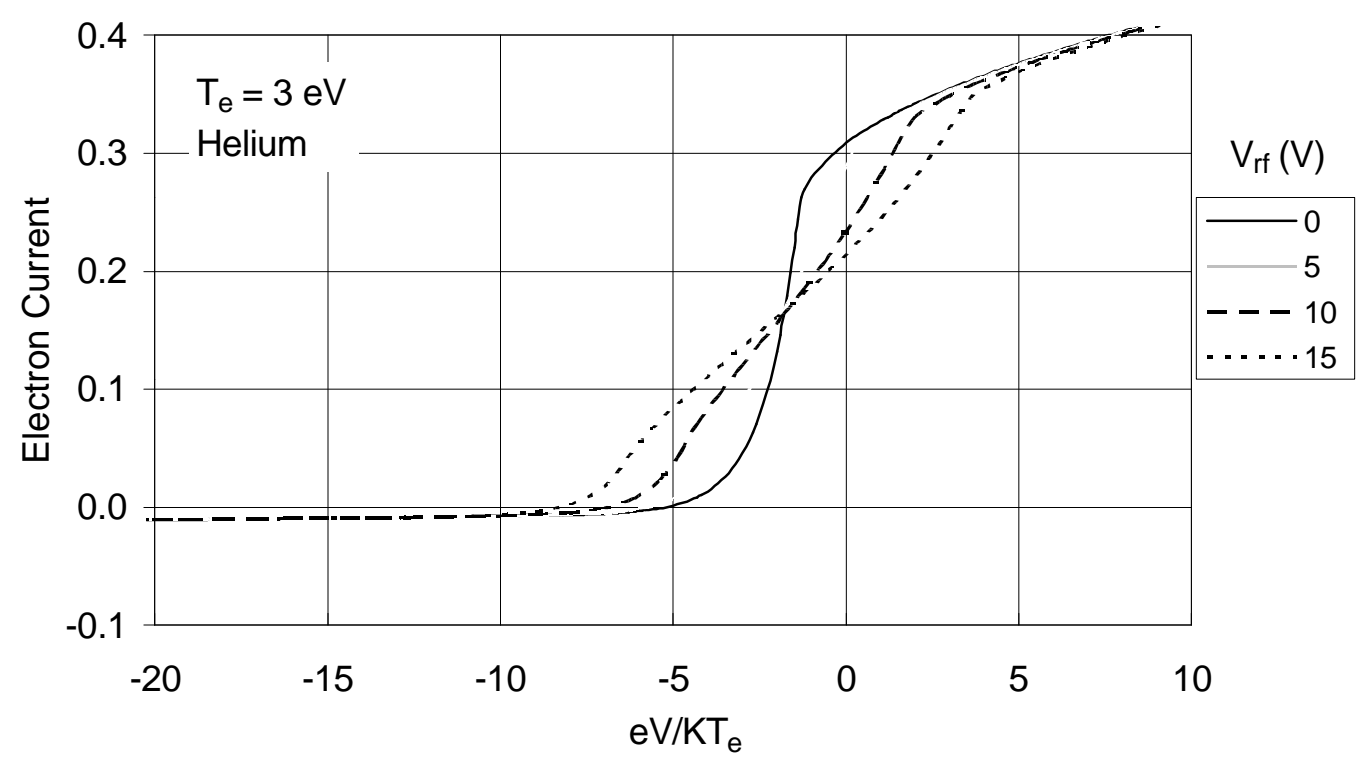

(b)

Fig. 10. (a) Normal $I-V$ characteristic for a $T_{\mathrm{e}}=3 \mathrm{eV}, T_{\mathrm{i}}=0$ Maxwellian plasma in a magnetic field. (b) The $I-V$ curve of (a) after distortion by RF oscillations of magnitude $V_{\mathrm{rf}}$ (c) Derived velocity distribution $f(v)$ vs. normalized probe voltage. 


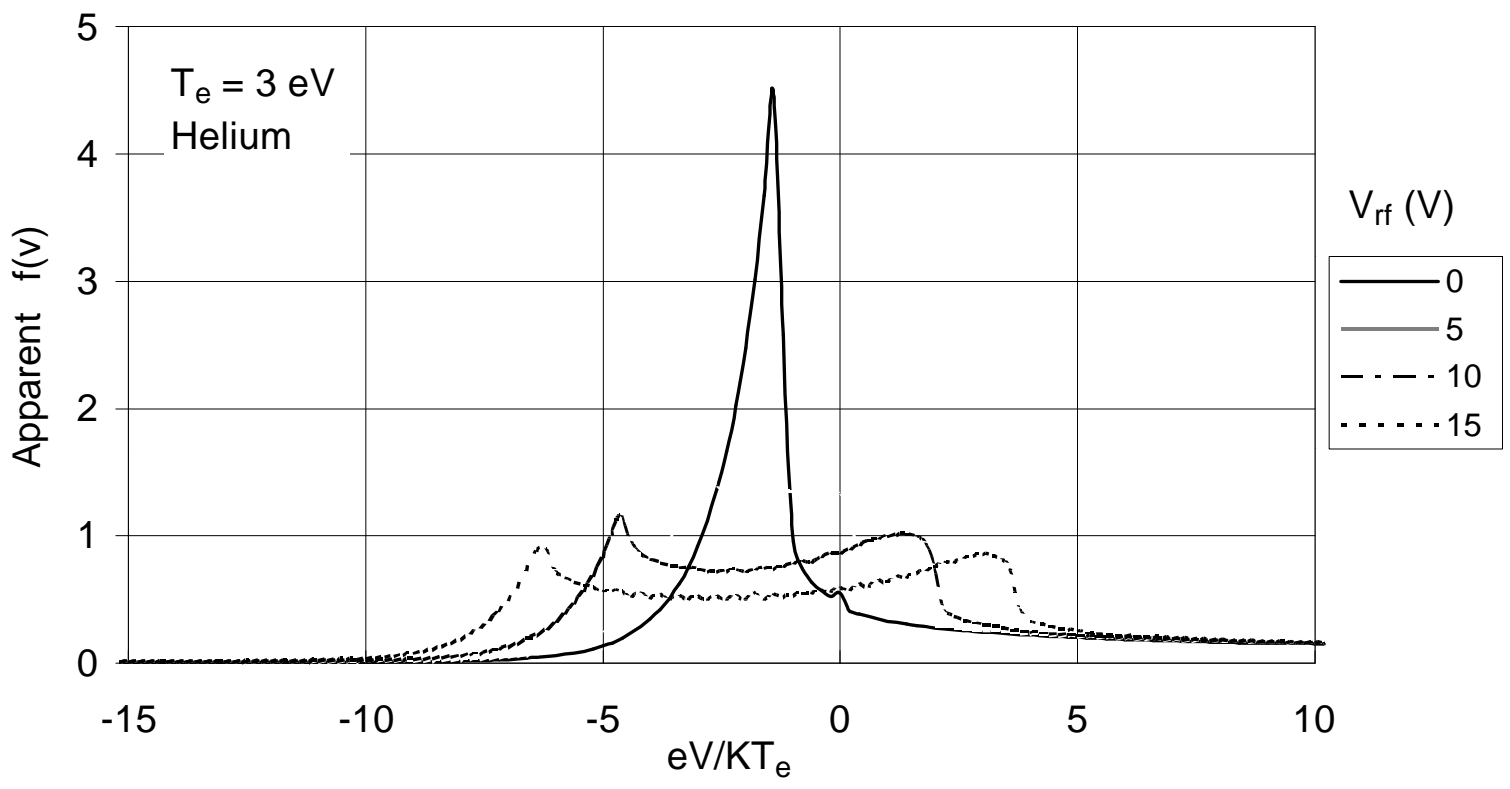

(c)

Fig. 10. (a) Normal $I-V$ characteristic for a $T_{\mathrm{e}}=3 \mathrm{eV}, T_{\mathrm{i}}=0$ Maxwellian plasma in a magnetic field. (b) The $I-V$ curve of (a) after distortion by RF oscillations of magnitude $V_{\mathrm{rf}}$ (c) Derived velocity distribution $f(v)$ vs. normalized probe voltage. 


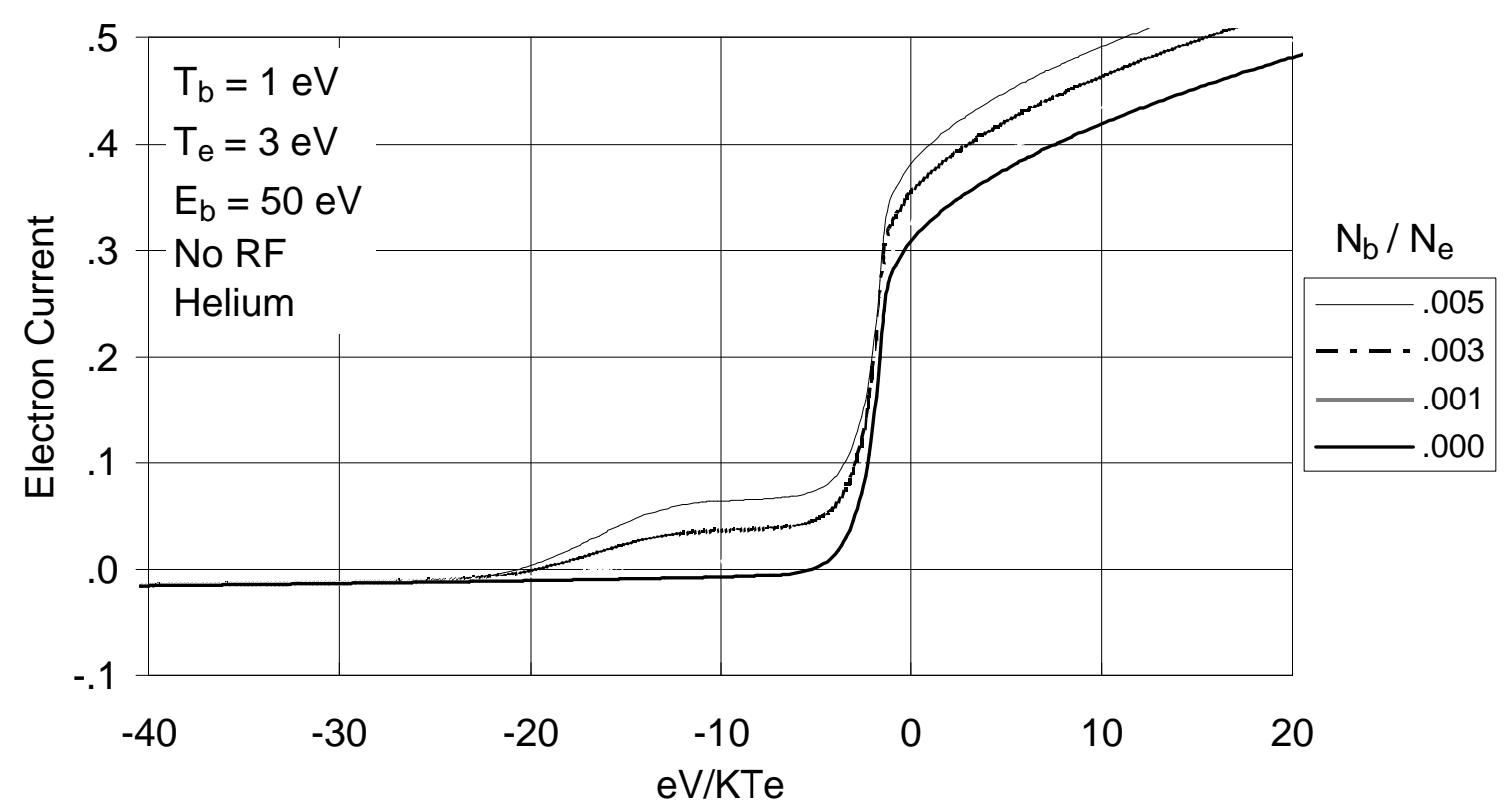

(a)

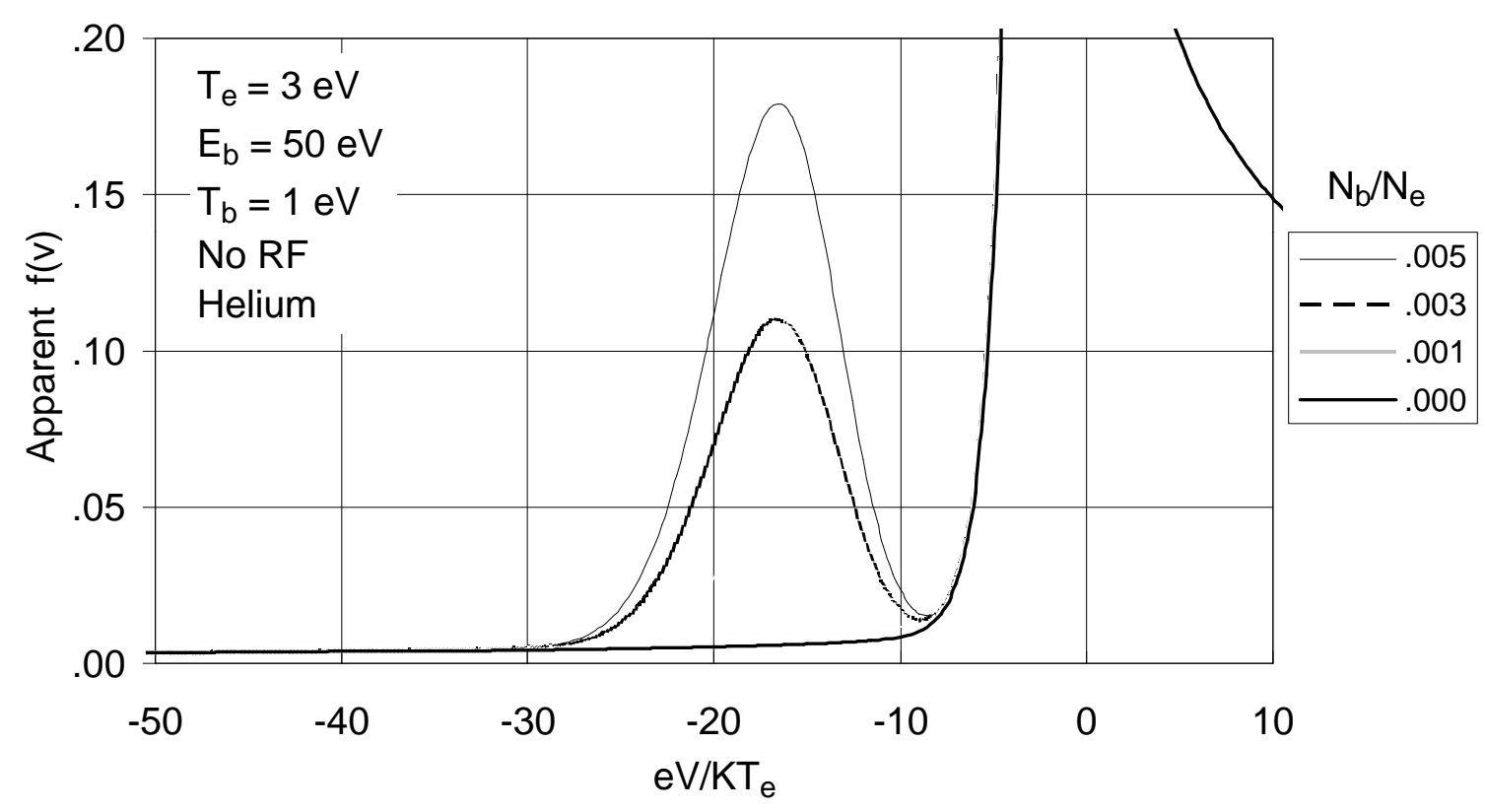

(b)

Fig. 11. (a) $\mathrm{I}-\mathrm{V}$ characteristics for a $3-\mathrm{eV}$ plasma with a $T_{\mathrm{b}}=1 \mathrm{eV}$ beam of various densities. (b) Apparent distributions $f(v)$ derived from (a). (c) Apparent $f(v)$ 's for a plasma with $50-\mathrm{eV}$ beams of various temperatures. The $I-V$ curves from which these were derived are not shown. (d) Apparent $f(v)$ 's for a plasma with beams of the same density but different energies. 


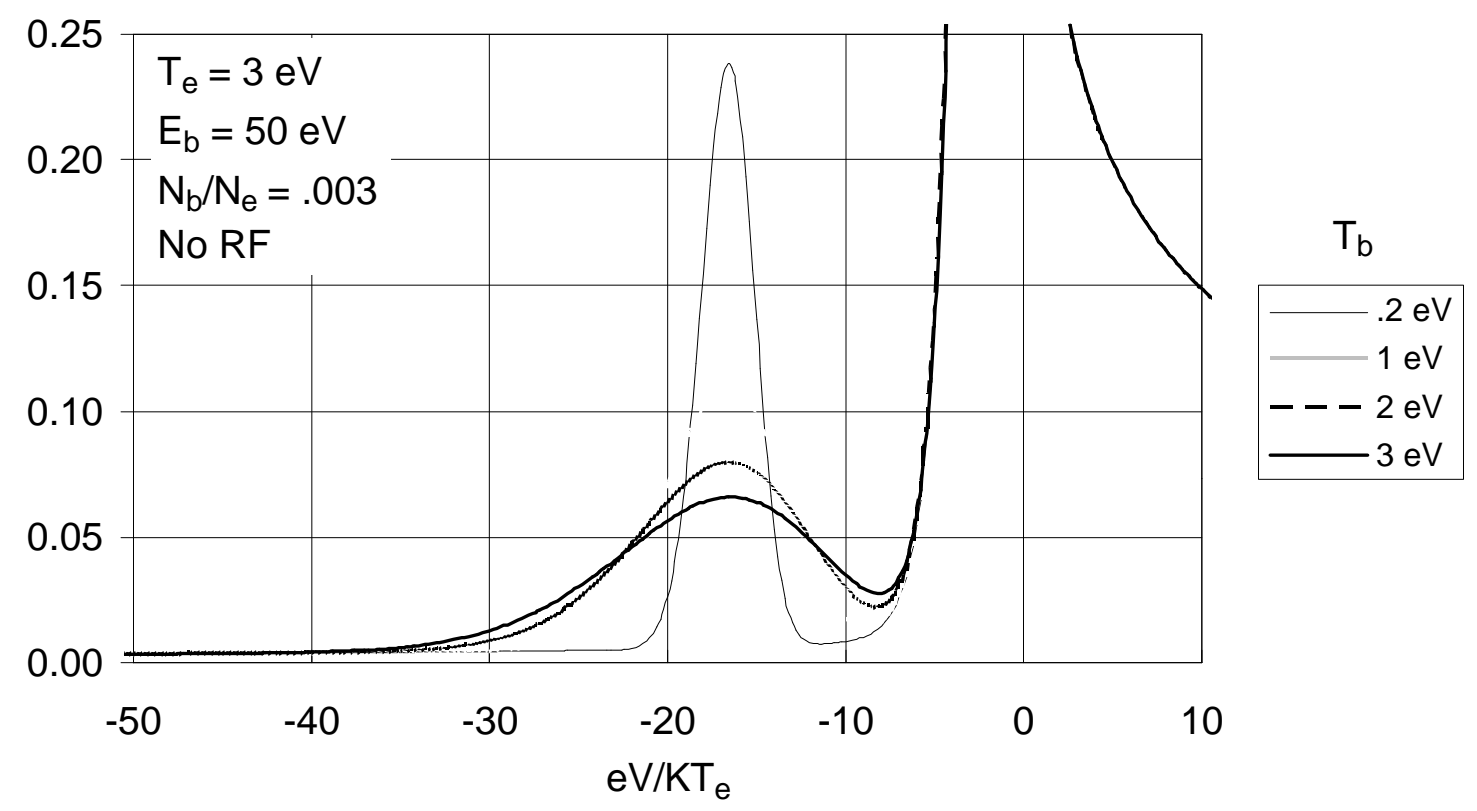

(c)

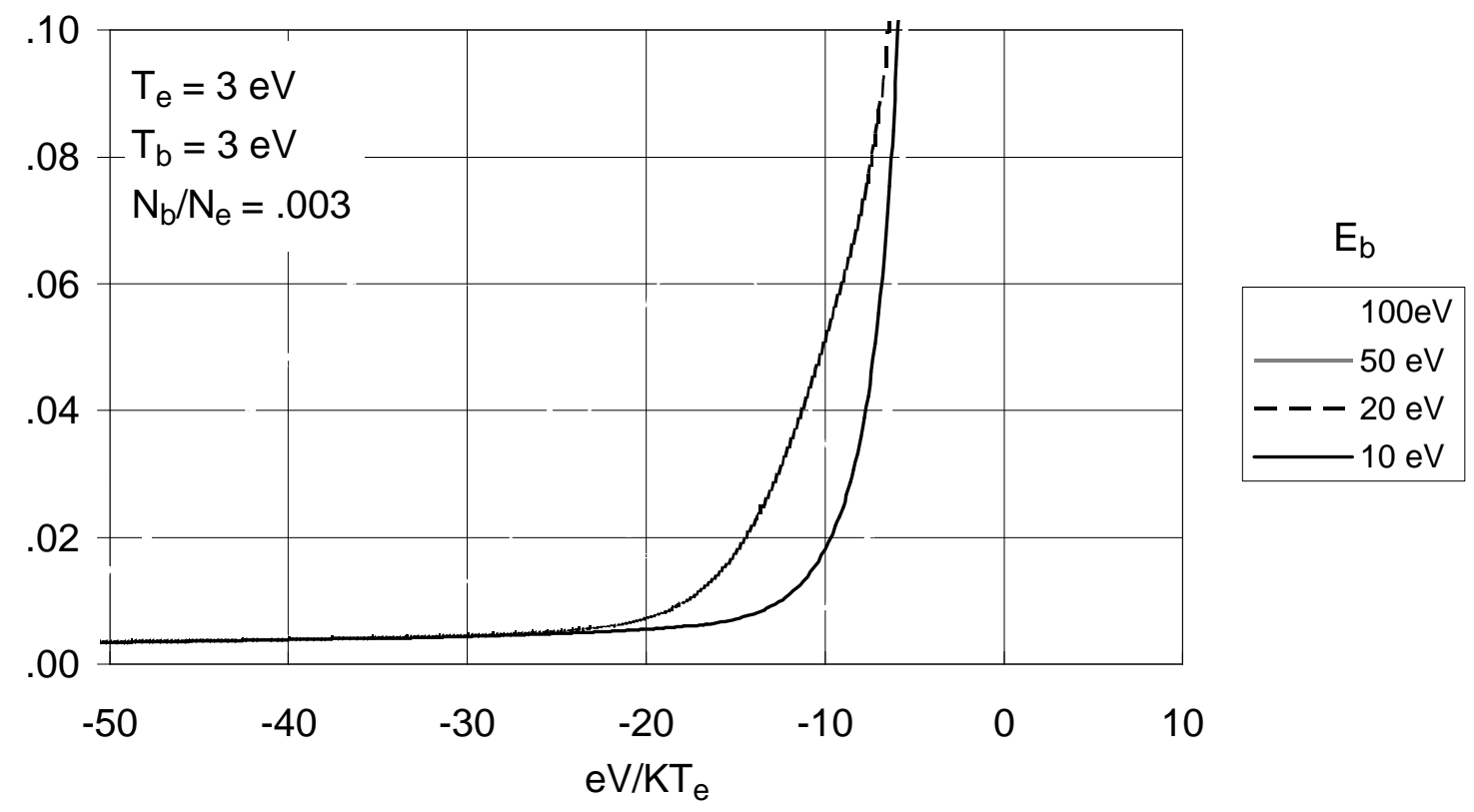

(d)

Fig. 11. (a) $\mathrm{I}-\mathrm{V}$ characteristics for a $3-\mathrm{eV}$ plasma with a $T_{\mathrm{b}}=1 \mathrm{eV}$ beam of various densities. (b) Apparent distributions $f(v)$ derived from (a). (c) Apparent $f(v)$ 's for a plasma with $50-\mathrm{eV}$ beams of various temperatures. The $I-V$ curves from which these were derived are not shown. (d) Apparent $f(v)$ 's for a plasma with beams of the same density but different energies. 


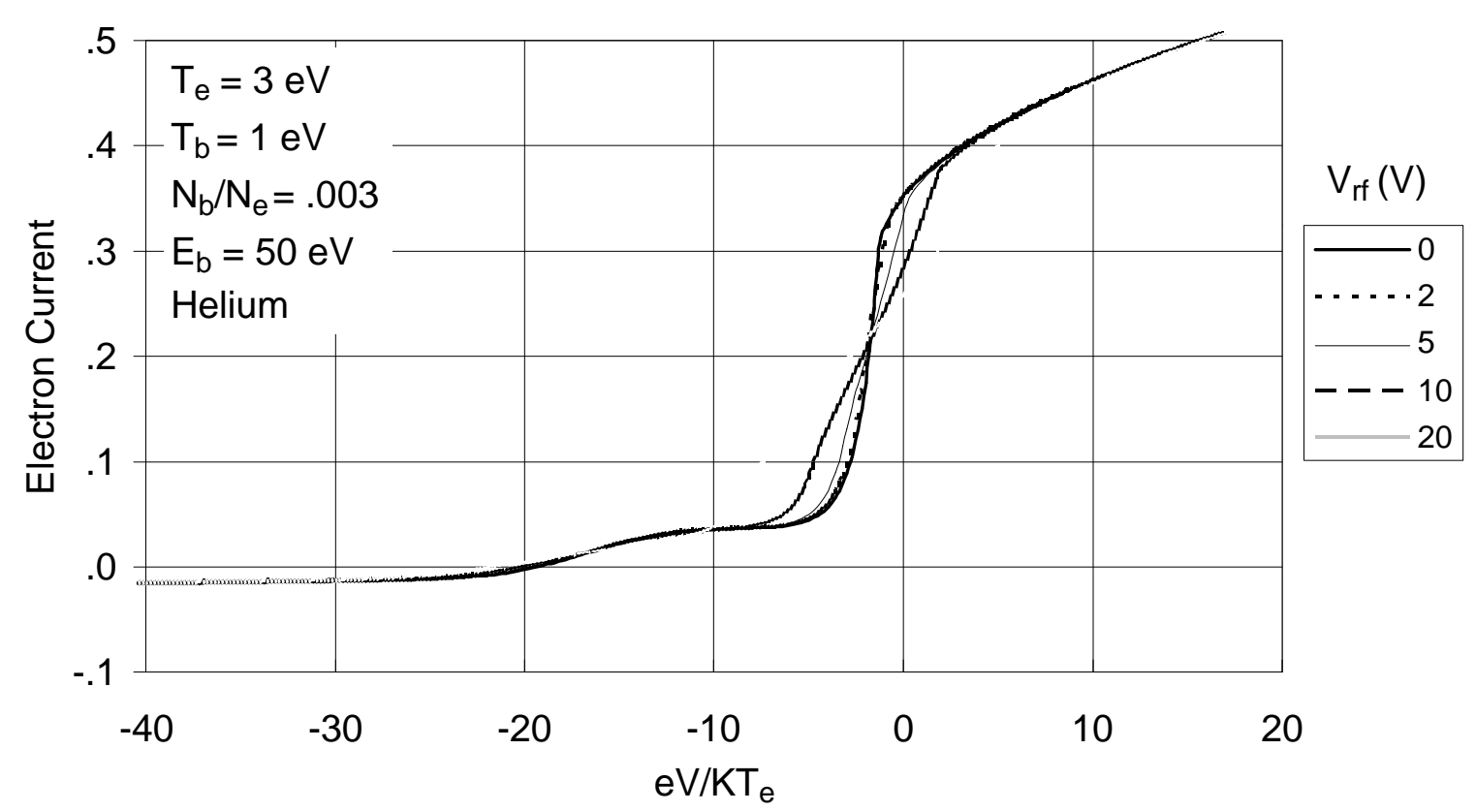

(a)

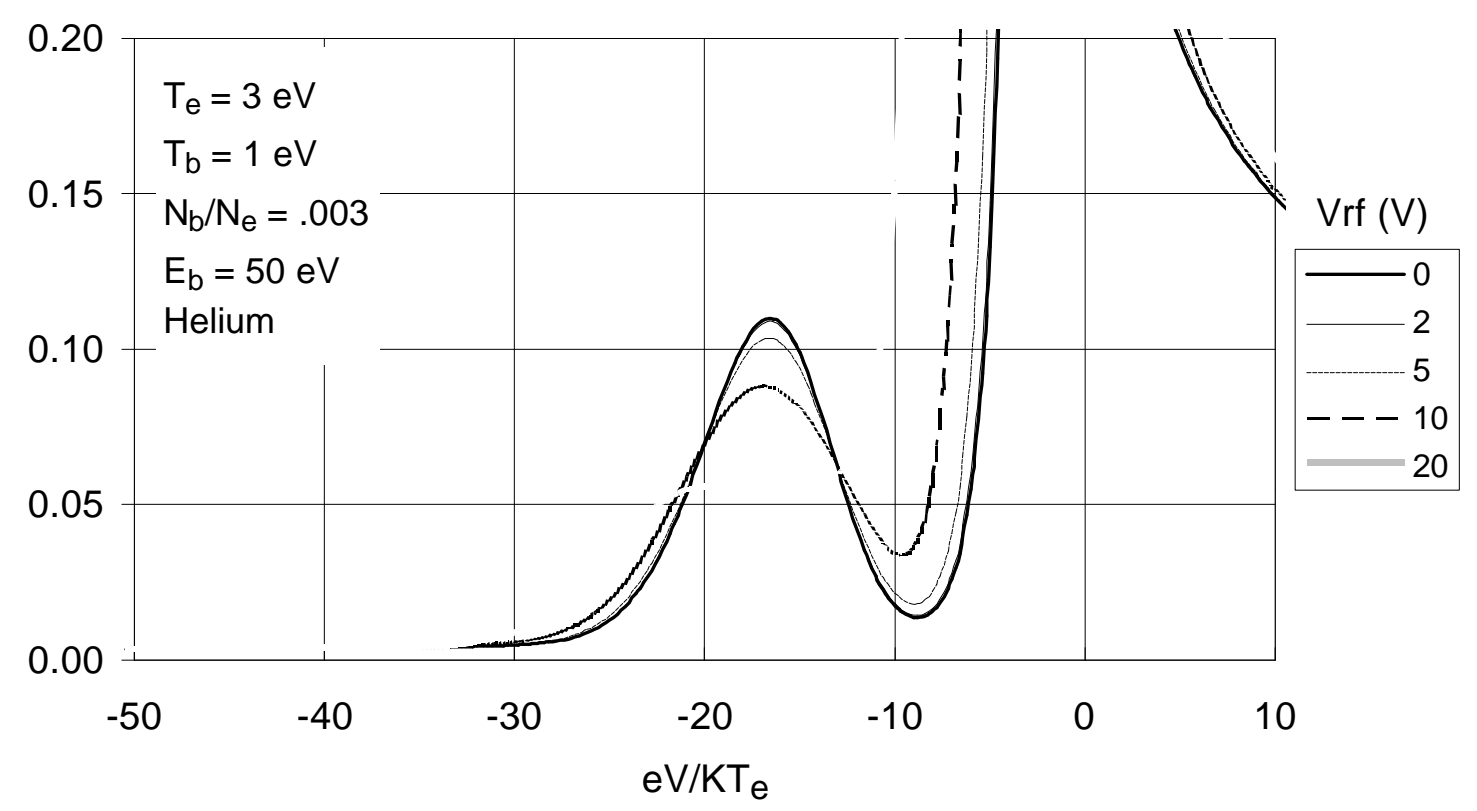

(b)

Fig. 12. (a) Probe characteristics for a plasma with a dc beam after distortion by various amounts of RF pickup. (b) Apparent distribution function in the region of the beam obtained by differentiation of the curves in (a). 


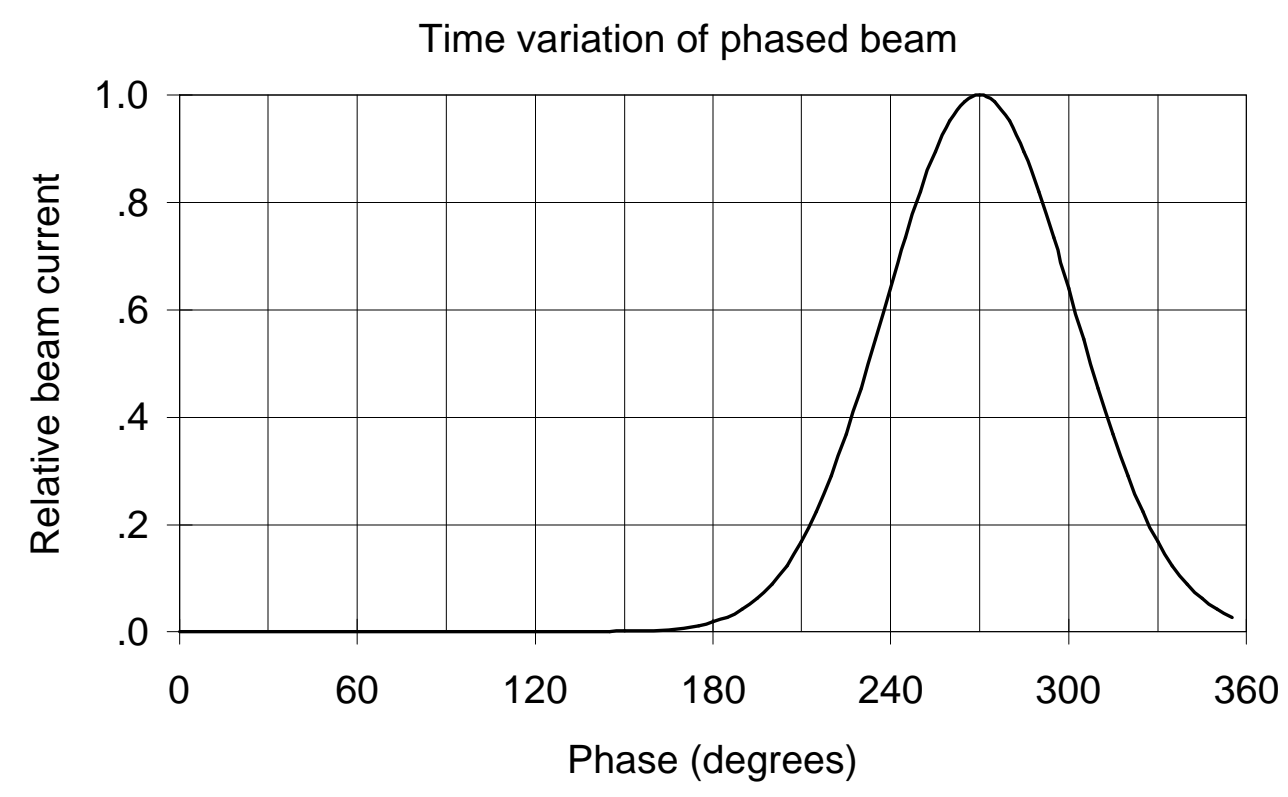

(a)

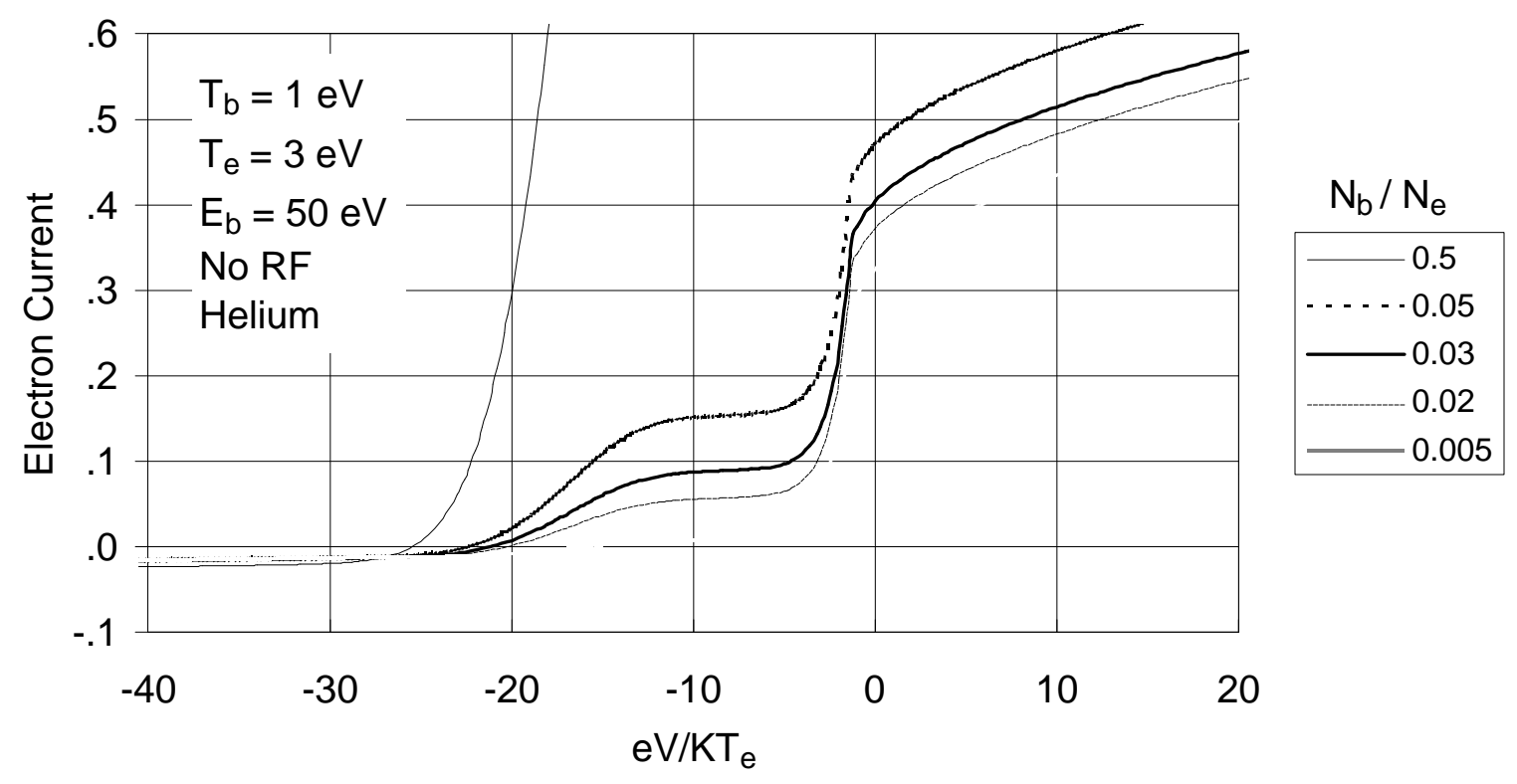

(b)

Fig. 13. (a) Time variation of phased electron beams used in the calculations. (b) Probe characteristics for a 3-eV plasma with a phased beam of various peak densities in the absence of RF pickup and of RF compensation circuitry. 


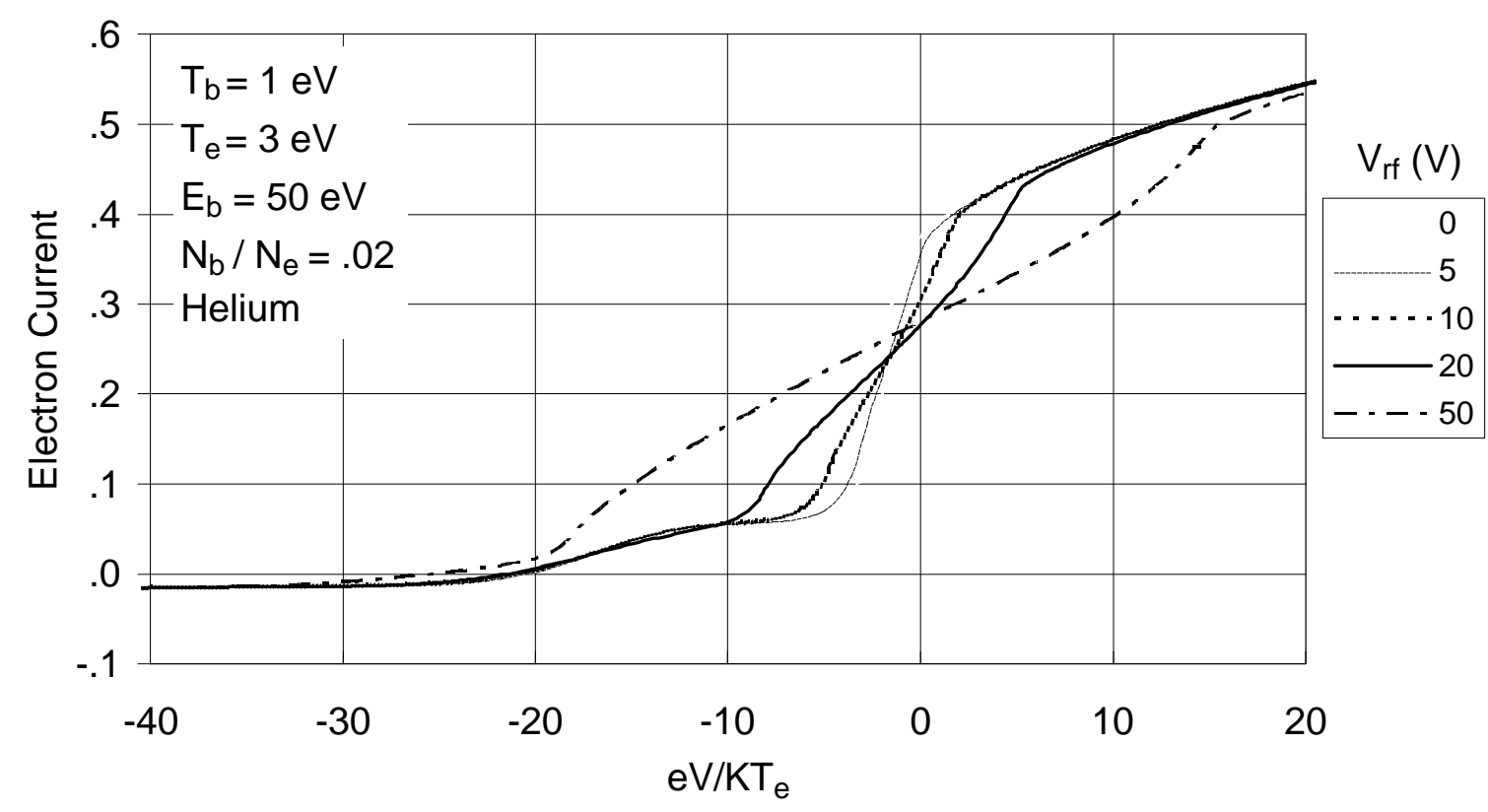

(a)

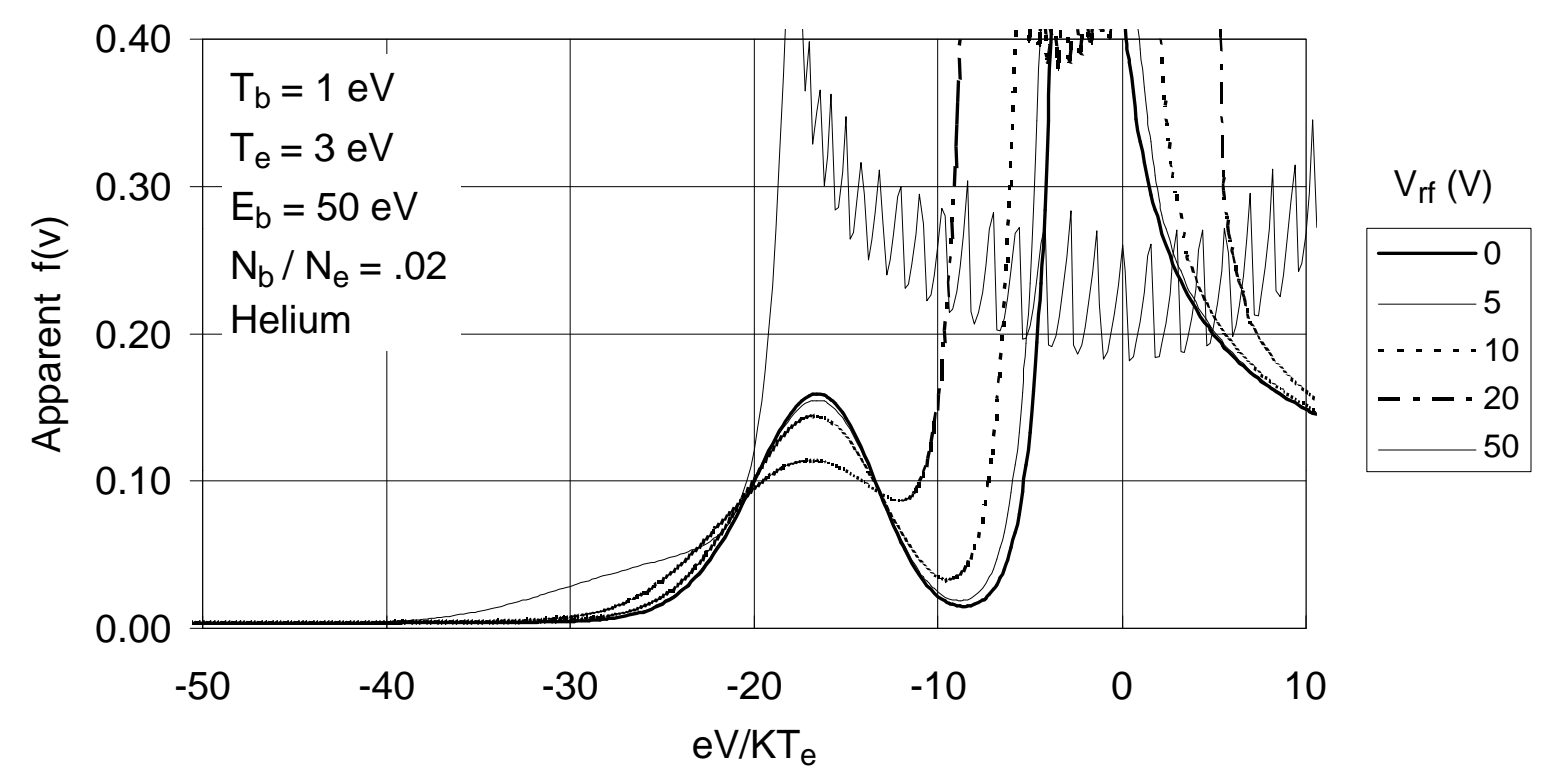

(b)

Fig. 14. (a) Uncompensated probe characteristics for a $3-\mathrm{eV}$ plasma with a phased beam and various levels of RF pickup. (b) Apparent distribution functions derived from (a). The rapid oscillations are due to the coarseness of the numerical grid and should be ignored. 


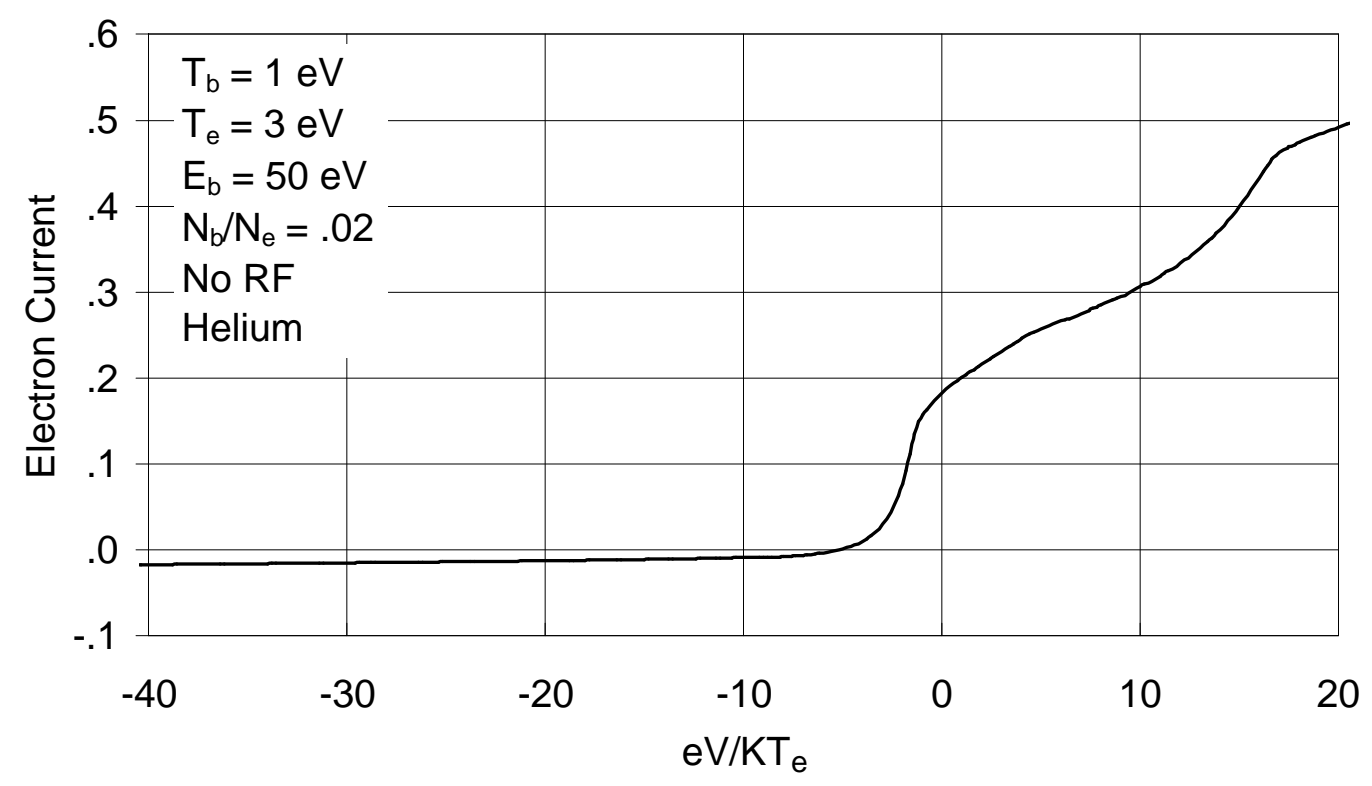

(a)

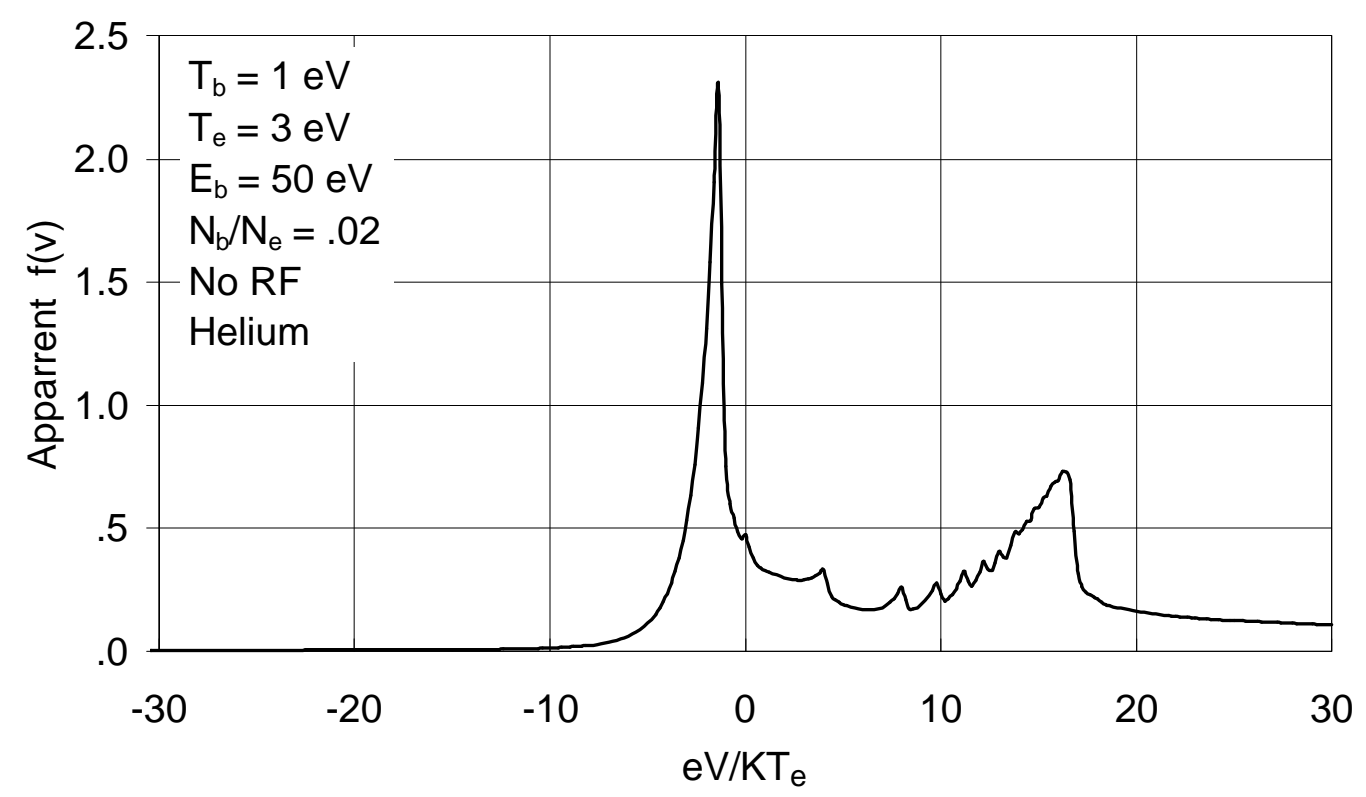

(b)

Fig. 15. (a) $I-V$ characteristic of a $V_{\mathrm{f}}$ - compensated probe in a $3-\mathrm{eV}$ plasma with a pulsed, $50-\mathrm{eV}$ electron beam. (b) Apparent $f(v)$ from the derivative of (a). 


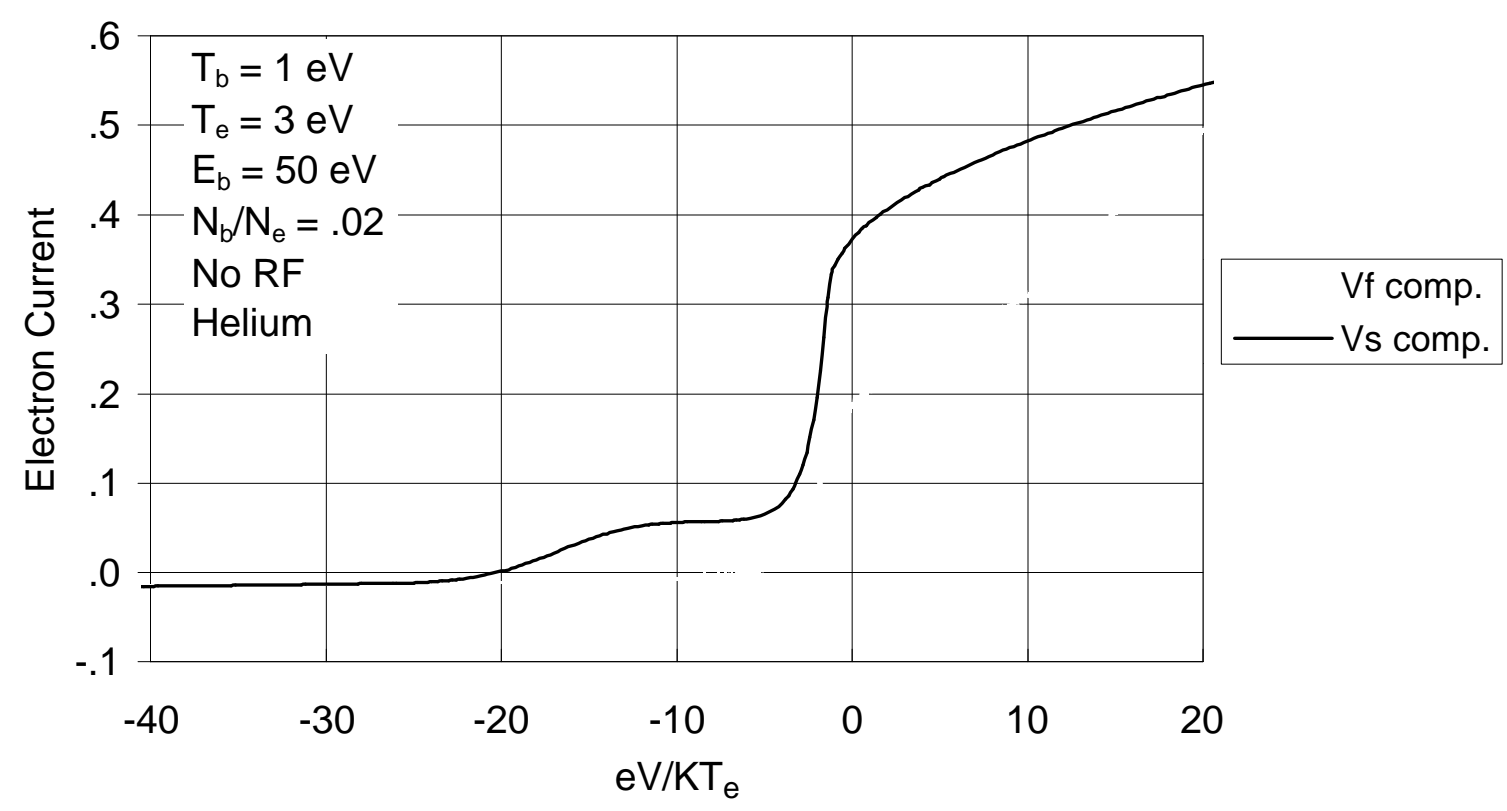

(a)

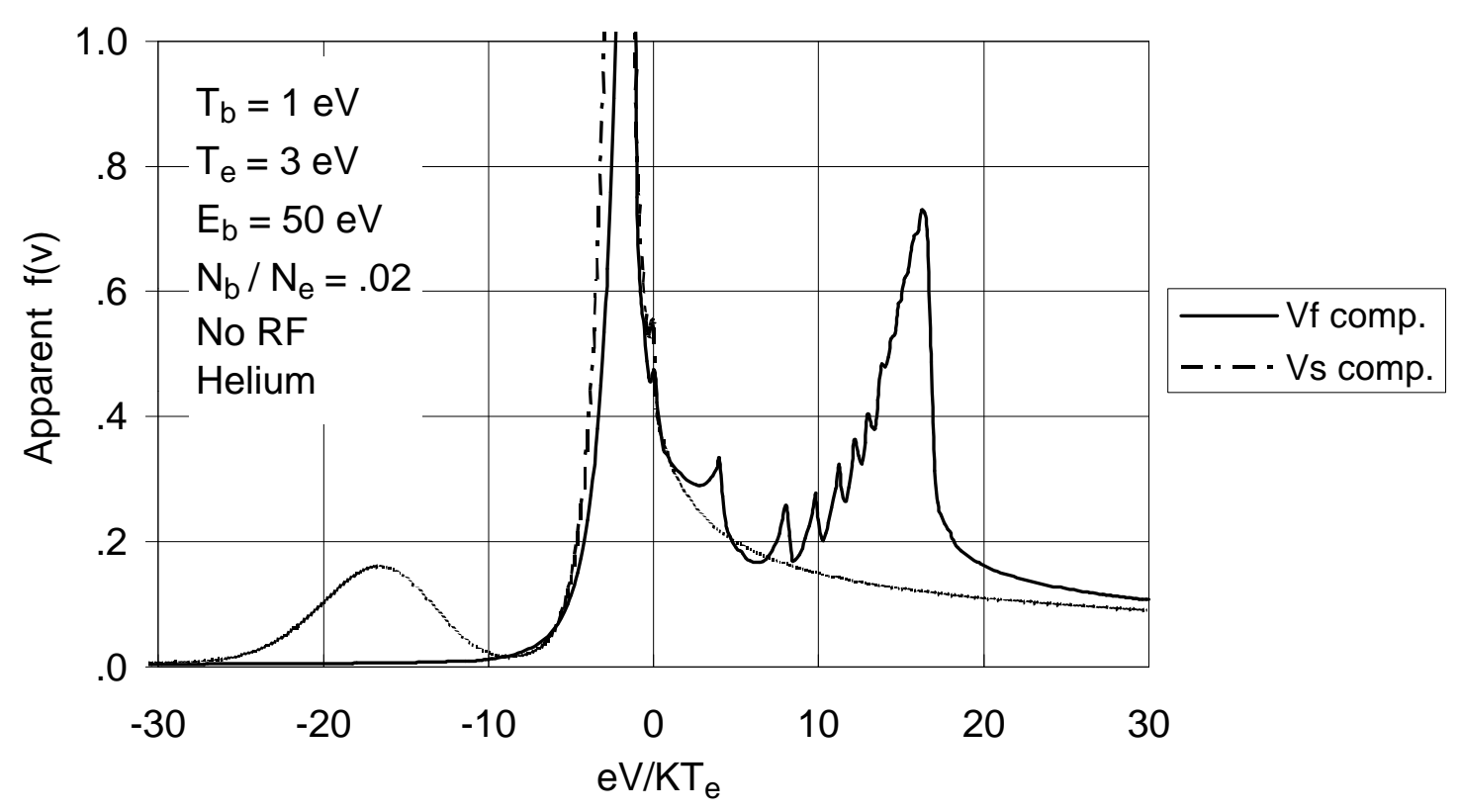

(b)

Fig. 16. (a) $I-V$ curves for probes driven to follow $V_{\mathrm{s}}$ and $V_{\mathrm{f}}$ in a plasma with a phased beam. (b) Apparent $f(v)$ 's derived from (a). 


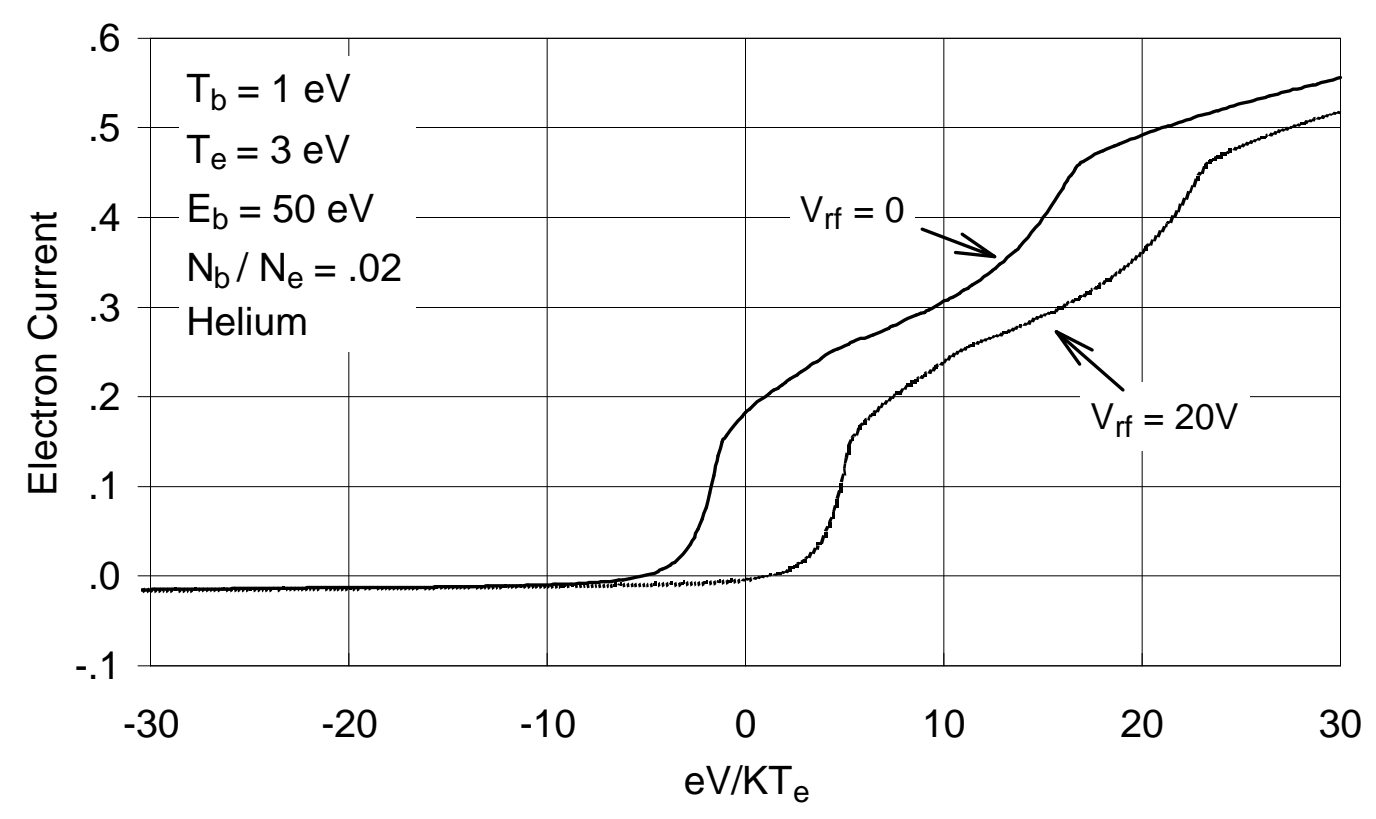

(a)

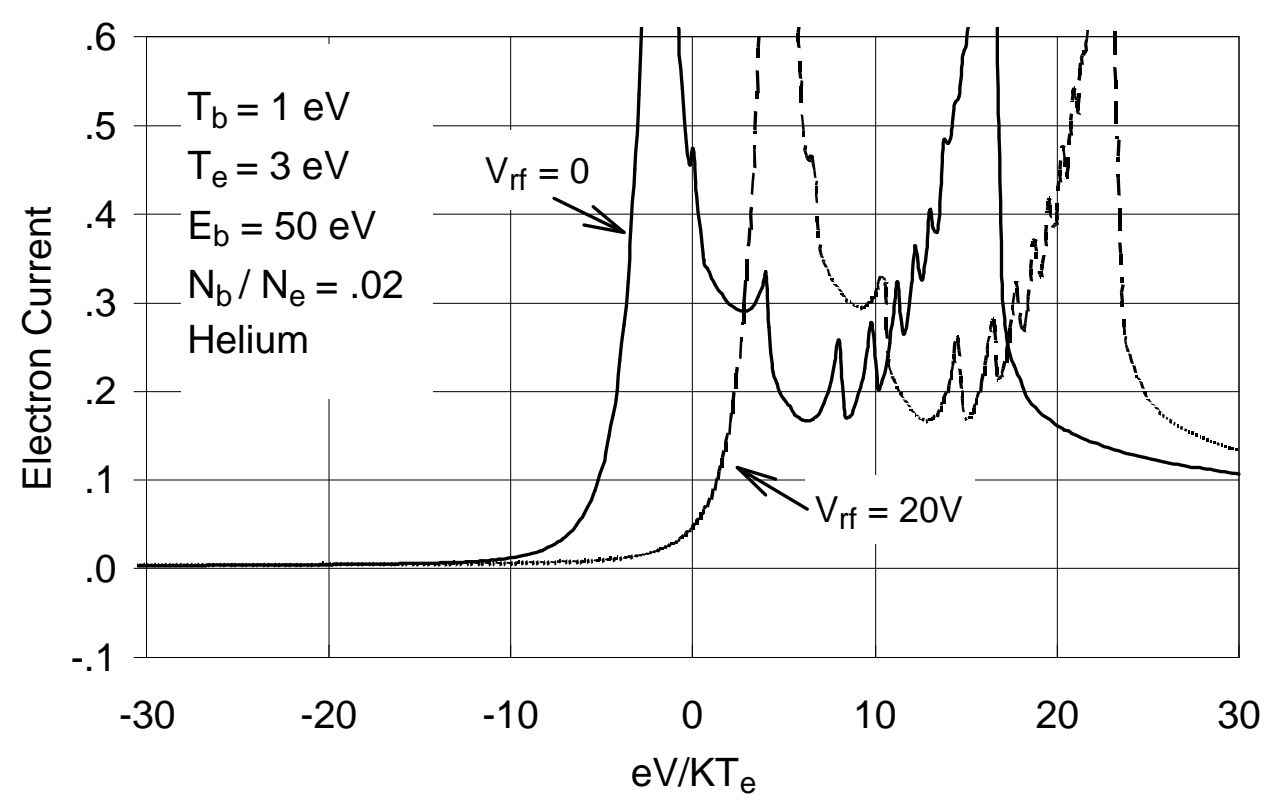

(b)

Fig. 17. (a) $I-V$ characteristics for $V_{\mathrm{f}}$-compensated probes in a plasma with a phased beam, with $V_{\mathrm{rf}}=0$ and $20 \mathrm{~V}$. (b) Apparent $f(v)$ 's derived from (a). 


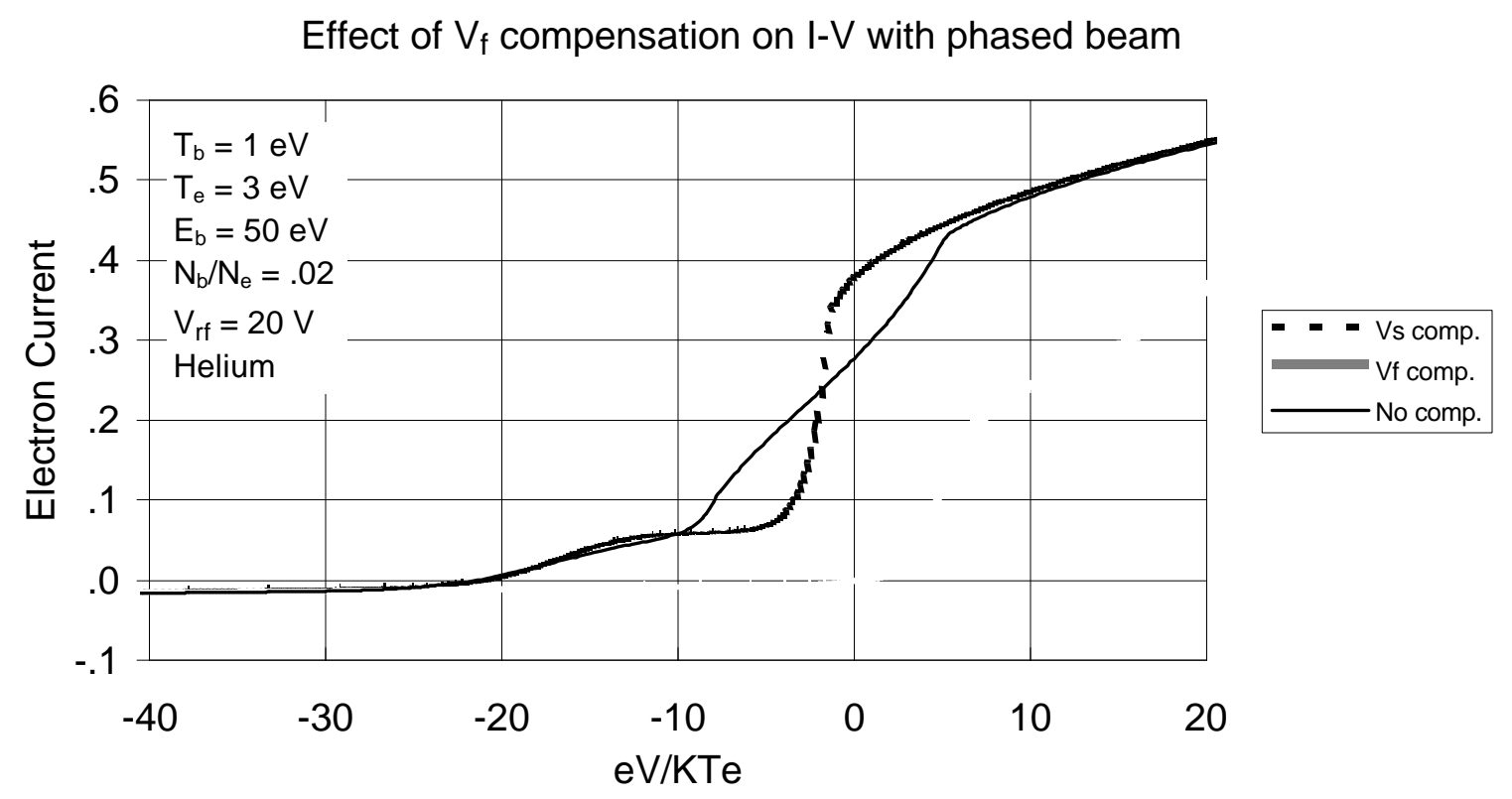

Fig. 18. $I-V$ characteristics for a probe in a plasma with a phased beam and $V_{\text {rf }}$ $=20 \mathrm{~V}$, for cases of no RF compensation, $V_{\mathrm{f}}$-compensation, and ideal $V_{\mathrm{s}^{-}}$ compensation. 